\title{
Article
}

\section{Modeling Quantum Dot Systems as Random Geometric Graphs with Probability Amplitude-Based Weighted Links}

\author{
Lucas Cuadra $^{1,2, *(\mathbb{D})}$ and José Carlos Nieto-Borge ${ }^{2}$ (D) \\ 1 Department of Signal Processing and Communications, University of Alcalá, 28801 Alcalá de Henares, Spain \\ 2 Department of Physics and Mathematics, University of Alcalá, 28801 Alcalá de Henares, Spain; \\ josecarlos.nieto@uah.es \\ * Correspondence: lucas.cuadra@uah.es
}

check for updates

Citation: Cuadra, L.; Nieto-Borge, J.C. Modeling Quantum Dot Systems as Random Geometric Graphs with Probability Amplitude-Based Weighted Links. Nanomaterials 2021, 11,375. https://doi.org/10.3390/ nano11020375

Academic Editor: Juan P. Martínez Pastor

Received: 21 December 2020

Accepted: 27 January 2021

Published: 2 February 2021

Publisher's Note: MDPI stays neutral with regard to jurisdictional clai$\mathrm{ms}$ in published maps and institutional affiliations.

\section{Copyright: $@ 2021$ by the authors. Li-} censee MDPI, Basel, Switzerland. This article is an open access article distributed under the terms and conditions of the Creative Commons Attribution (CC BY) license (https:// creativecommons.org/licenses/by/ $4.0 /)$.

\begin{abstract}
This paper focuses on modeling a disorder ensemble of quantum dots (QDs) as a special kind of Random Geometric Graphs (RGG) with weighted links. We compute any link weight as the overlap integral (or electron probability amplitude) between the QDs (=nodes) involved. This naturally leads to a weighted adjacency matrix, a Laplacian matrix, and a time evolution operator that have meaning in Quantum Mechanics. The model prohibits the existence of long-range links (shortcuts) between distant nodes because the electron cannot tunnel between two QDs that are too far away in the array. The spatial network generated by the proposed model captures inner properties of the QD system, which cannot be deduced from the simple interactions of their isolated components. It predicts the system quantum state, its time evolution, and the emergence of quantum transport when the network becomes connected.
\end{abstract}

Keywords: quantum dot; disorder array of quantum dots; probability amplitude; complex networks; spatial network; Random Geometric Graphs; quantum transport

\section{Introduction}

Conceptually, a quantum dot (QD) [1] is a "small" (less than the de Broglie wavelength), zero-dimensional (0D) nanostructure that confines carriers in all three directions in space [2-4], and exhibits a delta density of states (DOS), unlike those of quantum wells (2D nanostructure [5]) and quantum wires (1D) [2], as shown in Figure 1a. In particular, a (type I) semiconductor QD is a heterostructure [6] made up of a small island (generally 10$20 \mathrm{~nm}$ in size) of a semiconductor material ("dot material") embedded inside another with a higher bandgap ("barrier material" -BM-), which is able to confine both electrons and holes, as illustrated in Figure 1b). This creates discrete energy levels for carriers, and modifies both its electronic and optical properties [3]. The problem of manufacturing high densities of high-quality QDs is successfully addressed by using self-assembled QD (SAQD) [7] technologies. Figure 1c shows different SAQD growth modes. In the Stranski-Krastanow (SK) growth mode [8], the deposition of the dot material starts with the formation of a two-dimensional, very thin wetting layer (WL), and when a critical amount of strained dot material has been deposited, the formation of pyramidal QDs occurs to relax strain. In the Volmer-Weber (VW) mode, the QDs grow directly on the bare substrate [9]. Sub-monolayer (SML)-QDs [10] can be formed as a disk or as a spherical QD because the growth method consists of multilayer deposition with a fraction of a monolayer of dot material on the barrier matrix [8]. SML-QDs have several advantages over SK-QDs, such as a smaller base diameter $(5-10 \mathrm{~nm})$, higher dot density $\left(\sim 5 \times 10^{11} \mathrm{~cm}^{-2}\right)$, and better control of QD size $[10,11]$. 

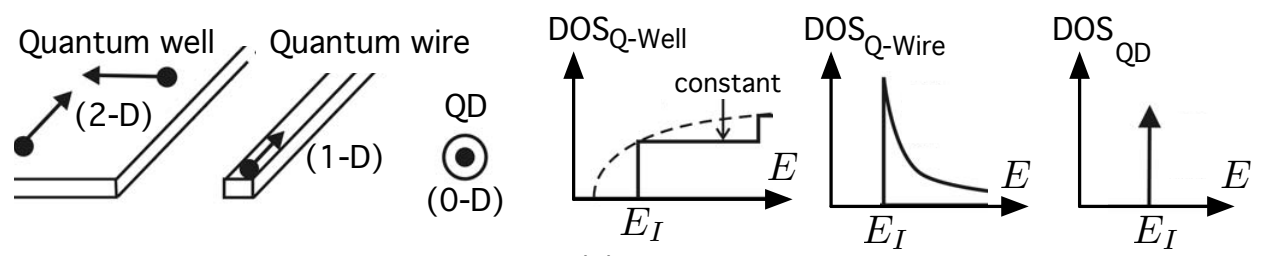

(a)

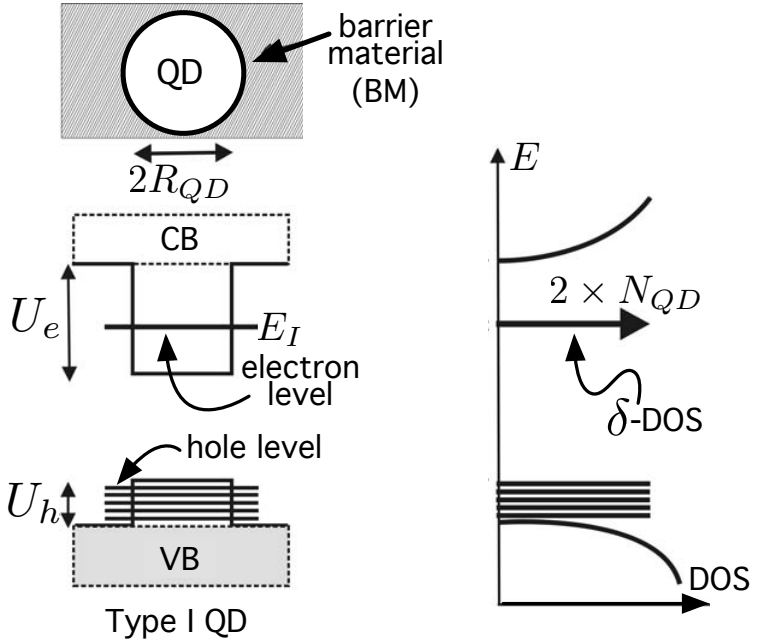

(b)

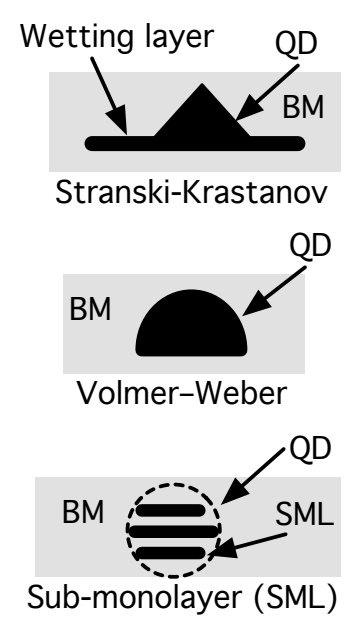

(c)

Figure 1. (a) Conceptual representation of nano-structures and their corresponding density of states (DOS) for quantum wells, quantum wires and quantum dots; (b) confinement potentials for electrons $\left(U_{e}\right)$ and holes $\left(U_{h}\right)$ in a type I QD (or simply, QD), and its corresponding density of states; (c) different classes of growth in self-assembled quantum dots. See the main text for details.

SAQD technologies help both research in Quantum Mechanics (QM) and manufacture novel devices. On the one hand, SAQD technologies assists in manufacturing high quality QDs for studying QM and exploring novel effects in electronics, photonics, and spintronics. For instance, SAQD-based devices help achieve single-electron charge sensing [12], entanglement between spins and photons [13,14], single-photon sources [15], or single-spin [16], and help also the control of Cooper pair splitting [17], spin transport [18], spin-orbit interaction [19], $g$-factor [20], and Kondo effect [21]. On the other hand, SAQD technologies allow for manufacturing high density of QDs, which are crucial for implementing optoelectronic devices such as QD-based light-emitting diodes (LEDs) [22], QD-memories [4,23], QD-lasers [24-27], QD-infrared photodetectors [8,28,29], and QD-solar cells [30]. A key point in these devices is that the position of carrier level(s) can be tuned by controlling the dot size [2], and, this, by modifying the growth conditions [6,10,11,31].

In this work, we model a system formed by QDs as a special graph in the effort of exploring electron transport. Our approach could be considered as belonging to Complex Networks (CN) Science. CN have become a multidisciplinary research field [32-34] for studying systems with a huge amount of components that interact with each other. These range from artificial systems (such as power grids [35,36], or the Internet [37]) to natural systems (vascular networks [38], protein interactions [39], or metabolic networks [40]). More examples showing the feasibility of $\mathrm{CN}$ to study many other systems can be found in $[32,41,42]$ and the references therein. All these very different systems have in common that all of them can be described in terms of a graph [32]: a set of entities called nodes (or vertices) that are connected to each other by means of links (or edges). A node represents an entity (generator/load in a power grid [36], or a species in an ecosystem [43]), which is connected with others (linked by electrical cables in power grids, or related by trophic relationships in ecosystems). This way, any system can be encoded as a graph, a mathematical abstraction of the relationships (links) between the constituent units (nodes) 
of the system. In broad sense, CN science models not only the structure (topology), but also some dynamic phenomena such as information spreading [44], epidemic processes (both biological [45], and artificial viruses [46]) or cascading failures [47,48]. These are very common in large engineered networks: wireless sensor networks [49], Internet [50], power grids [35,51,52], or transportation networks [53]. Some of these CN, such as transportation networks, are networks in which the nodes are spatially embedded [54] or constrained to locations in a physical space with a metric. For many practical applications, the space is the two-dimensional space and the metric is the Euclidean distance $d_{E}$. This geometric constraint usually makes the probability of having a link between two nodes decrease with the Euclidean distance [53]. This particular subset of networks are called spatial networks (SN) $[53,55]$ or spatially embedded CN [56]. A particular class of SN are Random Geometric Graphs (RGGs) [57] in which $N$ nodes are uniformly distributed over the unit square, while the link between two nodes $i$ and $j$ is formed if the Euclidean distance $d_{E}(i, j)<r$, a given model parameter. RGGs have been successfully used to model wireless sensor networks [58] and ad hoc networks [59] in which $r$ is related to the range of the radio devices.

The previous paragraph has shown that $\mathrm{CN}$ science has been applied to a broad variety of macroscopic, "classical" (non-quantum) systems. As will be shown in our review of the current state of the art in Section 2, CN science has also been used to study quantum nanosystems, although to a much lesser extent and not with the RGG approach that we will show throughout this paper.

The purpose of our work is to propose a particular class of RGG whose links have weights computed according to QM with the aim of studying electron transport in a system of disordered QDs like the one in Figure 2a. It consists of a number of $N$ QDs-for instance, a layer of SAQDs [6], which produce finite quantum confinement potentials (QCPs), randomly distributed in the physical metric space, $\mathbb{R}^{2}$, characterized by the Euclidean distance, $d_{E}$.

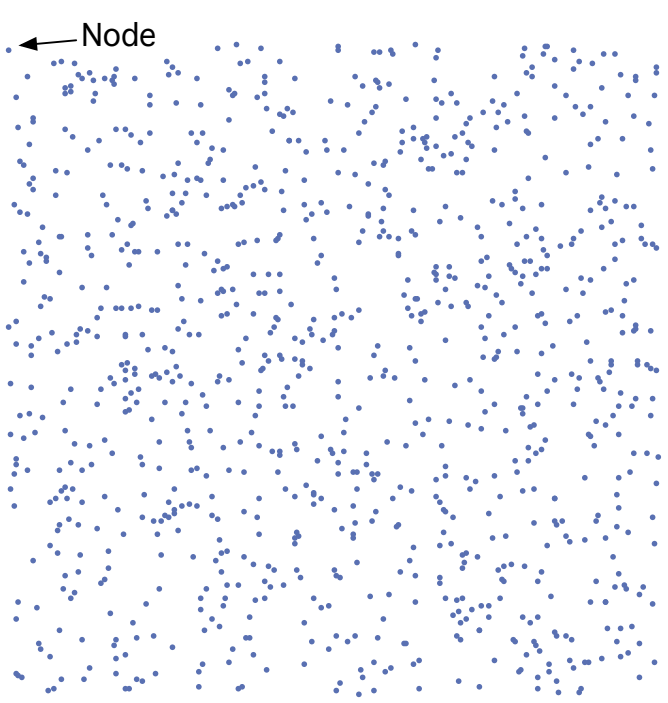

(a)

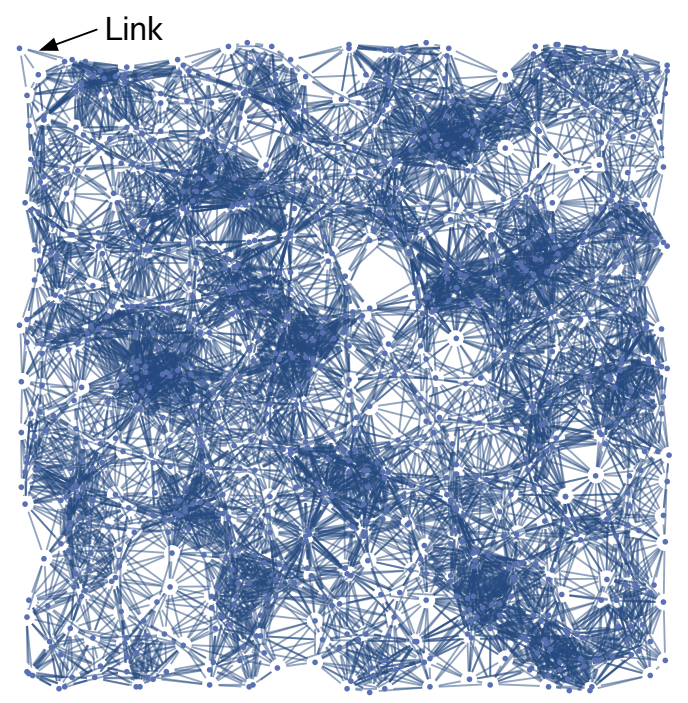

(b)

Figure 2. (a) Random distribution of QDs in $\mathbb{R}^{2}$. Each $Q D$, represented by a node, causes a quantum confinement potential. (b) A link between two nodes is allowed if and only if the electron wavefunctions at such nodes have non-zero overlap. Any link has a weight that is quantified by such overlap. See the main text for details.

In our model, any QD causing a QCP in Figure 2a is represented by a node. To understand how links have been generated in Figure $2 b$, it is convenient to have a look at Figure 3. On its left side, we have represented, for illustrative purposes, two of these QDs, labeled $i$ and $j$. For the sake of clarity, we have assumed that each QD 
has radius $R_{Q D}$. Their centers are separated by a Euclidean distance $d_{E}(i, j)$. As shown in Figure 3a, we have also represented an $\xi$ axis that passes through the centers of both QDs. The $\xi$ axis will assist us in clearly representing the associated concepts in Figure $3 b, c$. Figure $3 \mathrm{~b}$ shows the corresponding finite QCP caused by a QD along $\xi$ axis: $V(\xi)=-V_{C}$ (inside the QD) and $V(\xi)=0$ (otherwise). Since the $\mathrm{QCP}$ is finite, there is a part of the electron wavefunction that spreads outside the QD, as qualitatively shown in Figure 3c. Note that the QDs are close enough that the electron wavefunctions overlap. According to $\mathrm{QM}$, the electron, $e^{-}$, is in both QDs with a probability amplitude as the one in Figure 3c. The electron can tunnel from one QD to the other. We model this quantum phenomenon by forming a link between nodes $i$ and $j$ (Figure 3d). We will show throughout the paper that the link weight $w_{i j}$ between two nodes $i$ and $j$ depends on the extent to which the electron wave-functions in both nodes overlap. Quite often, however, there are electron wave-functions in sufficiently remote QDs that do not overlap at all. Regarding this, we have represented in Figure 3e two nodes that are so far apart that their corresponding wave-functions do not overlap (Figure 3g). Thus, an electron that is in node $i$ at the initial time $t=0$ will remain localized in that node even if $t \rightarrow \infty$. We model this QM result through the absence of a link $\left(w_{i j}=0\right)$, as shown in Figure $3 \mathrm{~h}$ (not connected nodes).

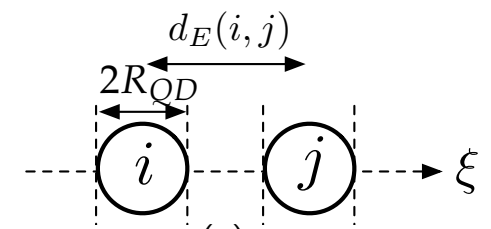

(a)

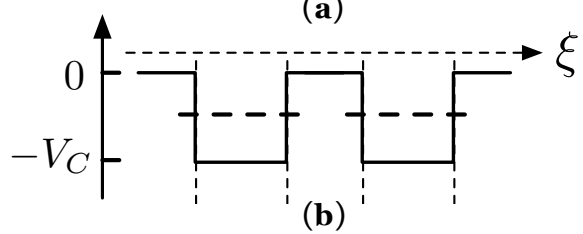

(b)

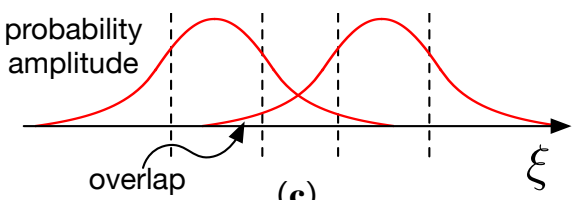

(c)

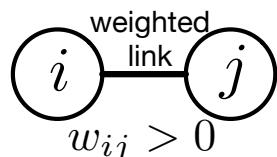

(d)

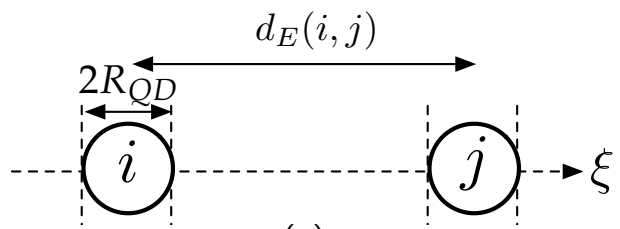

(e)

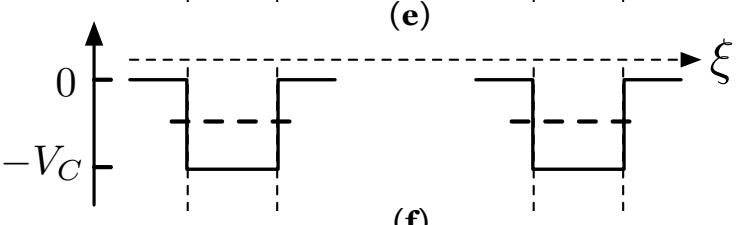

(f)

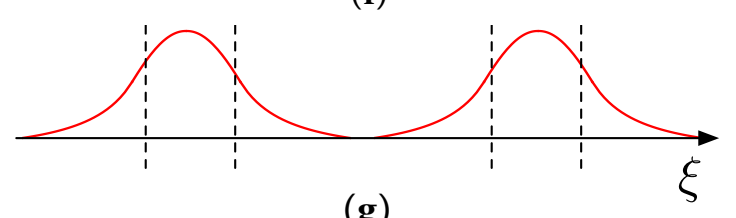

$(\mathbf{g})$

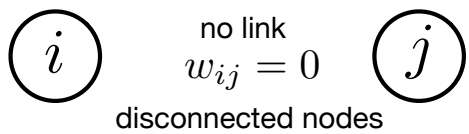

(h)

Figure 3. (a) Two of the QDs as those in Figure 2a. We consider QDs with radius $R_{Q D}$ and whose centers are separated by a Euclidean distance $d_{E}(i, j) . \xi$ represents an axis that passes through the center of both QDs. Along the $\xi$-axis, we have represented in $(\mathbf{b})$ the corresponding QCP $\left(-V_{C}\right.$ inside the node, 0 otherwise) and the electron probability amplitudes (c). An electron can be in both QDs with the represented probability amplitude. (d) We model this with a link whose weight $w_{i j}$ is given by the overlap. (e) Opposite case in which the QDs are so far apart (f) that there is no overlap (g) and link formation is not allowed (h).

Our main result is that the proposed RGG is able to capture inner properties of the complex quantum system: it predicts the system quantum state, its time evolution, and the emergence of quantum transport (QT) as the QD density increases. In fact, QT efficiency exhibits an abrupt change, from electron localization (no QT) to delocalization (QT emerges). This is an electron localization-delocalization transition that has also observed in [60].

Our proposal could have potential application not only in improving the efficiency of QD-based optoelectronic devices (LEDs, solar cell, lasers, etc.) that make use of SAQD 
layers but also in single, huge macromolecules (light-harvesting molecules [61]) to study the quantum transport (energy, charge) between specific areas of their structures.

The rest of this paper has been structured as follows. After reviewing of the current state of the art in Section 2, Section 3 briefly introduces the QD system that we want to study, while Section 4 explains our RGG proposal. The experimental work in Section 5 allow for predicting inner features of the system such as the system quantum state, its time evolution, or the emergence of quantum transport. Section 6 discusses potential applications, strengths, and weaknesses of the proposed method. Finally, Section 7 completes the paper. Appendix A lists the symbols used in this work.

\section{Current State of the Art}

There are basically two approaches that combine $\mathrm{CN}$ and QM concepts [62]. The first one applies concepts inspired by QMs to better study CN. For instance, Ref. [63] proposes a way to navigate complex, classical (non-quantum) networks (such as, the Internet) based on quantum walks [64] (the quantum mechanical counterpart of classical random walks [65]). More examples belonging to this first framework can be found in [62] and in the references therein. The second approach, in which the present article can be included, is based on applying $\mathrm{CN}$ concepts to explore nanosystems, which are governed by the laws of QM [66] and not by those of classical physics. A representative instance is the system studied in [67]: any atom trapped in a cavity is represented by a node, while the photon that the two atoms (nodes) exchange is encoded by a link between them. This and other papers have in common the fact of studying quantum properties on networks using quantum walks. This is because the quantum dynamics of a discrete system can be re-expressed and interpreted as a single-particle quantum walk [68,69]. This is the reason why quantum walks have been used to study the transport of energy through biological complexes involved in light harvesting in photosynthesis [70]. Quantum walks have also been used to explore transport in systems described by means of $\mathrm{CN}$ with different topologies [71,72]. Specifically, continuous-time quantum walks (CTQW)—a class of quantum walks on continuous time and discrete space [64] — have been used extensively to study quantum transport (QT) on CN [72], and will also be used in our work. There are several works that have studied QT over regular lattices [72-74], branched structures $[75,76]$ (including dendrimers [76]), fractal patterns [77], Husimi cacti [78], Cayley trees [79], Apollonian networks [80], scale-free networks [81], small-world (SW) networks [82] and start graphs [83,84], leading to the conclusion that QT differs from its classical counterpart. Having a quantitative measure of the efficiency of QT in a CN has been found to be important for practical and comparative purposes. In this regard, Ref. [85] has recently found bounds that allow for measuring the global transport efficiency of $\mathrm{CN}$, defined by the time-averaged return probability of the quantum walker. QT efficiency can undergo abrupt changes, and can have transitions from localization (no QT) to delocalization (QT appears). In this respect, the authors of [60] have studied localization-delocalization transition of electron states in SW networks. The SW feature is interesting because it makes it easy to navigate a network since SW networks exhibit a relatively short path between any pair of nodes [86,87]: the mean topological distance or average path length $\ell$ is small when compared to the total number of nodes $N$ in the network $(\ell=\mathcal{O}(\ln N)$ as $N \rightarrow \infty)$. The usual technique of rewiring [86] or adding links [88] in macroscopic, non-quantum $\mathrm{CN}$ to create SW networks have also been extended to quantum system $[82,84]$ to enhance QT. In [82], SW networks have been generated from a one-dimensional ring of $N$ nodes by randomly introducing $B$ additional links between them. The quantum particle dynamics has been modeled by CTQWs, computing the averaged transition probability to reach any node of the network from the initially excited one. Finally, the strategy of adding new links have been explored in star networks with the aim of enhancing the efficiency of quantum walks to navigate the network [84]. Please note that all of these key works have focused their research from the viewpoint of the topological properties. In particular, the topological (geodesic) distance between two nodes $i$ and $j, d(i, j)$, is the length of the 
shortest path (geodesic path) between them, that is, the minimum number of links when going from one node to the other [50]. The distance between two nodes $i$ and $j$ that are directly linked is $d(i, j)=1$, regardless of where they are located in physical space. We propose in the next paragraph to use the Euclidean distance for reasons that will be clearer later on.

\section{The QD System}

Let us consider a microscopic physical system, which is closed, and made of a set of $N$ QDs that are randomly distributed in a metric space as shown in Figure 2a. The position of each nanostructure in the metric space $\mathbb{R}^{2}$ is determined by a position vector $\mathbf{r}$. We consider a single electron (walker) freely tunneling among QDs (when allowed). An example of one-electron model is the tight-binding model [89], which has been used for both lattice and random networks.

Aiming to later numerically illustrate the results of studying this system using CN concepts, we are going to assume a set of hypotheses about the QCF that the QDs produce. These hypotheses will allow us to tackle the problem by using some well known QM results on each individual nanostructure, so that we will be able to then focus on exploring the complete system as a RGG.

With this in mind, and for reasons that will be clearer later on, we first assume that the single band effective mass equation of electrons in the envelope approximation [90] is a proper description of the dot and barrier bulk materials. This is because a QD size of 10 to $20 \mathrm{~nm}$ is much larger than the lattice constant of the material involved and, thus, it seems reasonable to consider that only the envelope part of the electron wave function is affected by the confinement potential. For the sake of clarity, we also assume that the QDs are identical (since in a closed system, energy is conserved, and the electron can only make transitions between QDs that have the same energy, that is, between QDs that have the same size [91]) and spherical with radius $R_{Q D}$. The center of any QD $i$ is given by a position vector $\mathbf{r}_{i}$ in the metric space. We assume that its associated QCP is spherically symmetric (depending only on the radial co-ordinate $r$ ), finite and "square":

$$
U_{C}(r)=\left\{\begin{array}{cl}
-V_{C} & , \text { if } r<R_{Q D} \\
0 & , \text { if } r>R_{Q D}
\end{array}\right.
$$

as shown in Figure 4a.

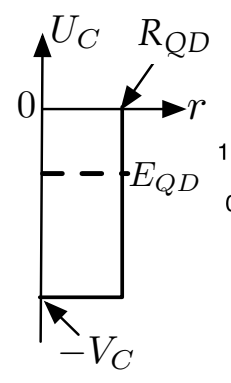

(a)

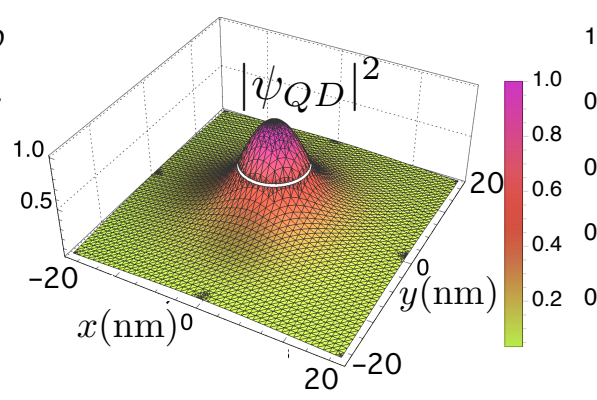

(b)

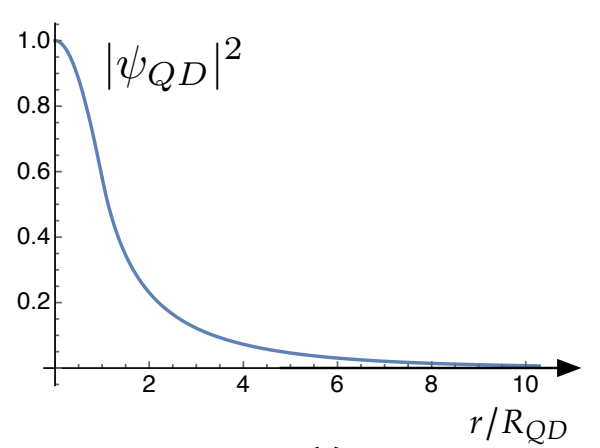

(c)

Figure 4. (a) Quantum confinement potential. $V_{C}$ is the depth of the potential well while $R_{Q D}$ is the radius of the QD producing $U_{C} . E_{Q D}$ is the energy of the bound state; (b) squared modulus of the corresponding wave function, $\left|\psi_{Q D}\right|^{2}$, in Cartesian coordinates; $(\mathbf{c})\left|\psi_{Q D}\right|^{2}$ as a function of the normalized radial coordinate, $r / R_{Q D}$.

The reason why we have made use of $U_{C}$ is that the time-independent Schrödinger's equation, which allows for computing both the electron wavefunction $\left(\psi_{Q D}\right)$ and its en$\operatorname{ergy}\left(E_{Q D}\right)$, 


$$
\widehat{H} \psi_{Q D}=E_{Q D} \cdot \psi_{Q D},
$$

can be solved analytically [91-93]. $\widehat{H}$ in Equation (2) is called the Hamiltonian operator and corresponds to the total energy of the system,

$$
\widehat{H} \equiv-\frac{\hbar^{2}}{2 m} \nabla^{2}+\widehat{V}
$$

where $\hbar$ is the reduced Planck constant, $m$ the electron mass, $\nabla^{2}$ is the the Laplace operator [94], and $\widehat{V}$ is the energy potential operator.

Thus, according to QM, the electron energy in the QCF (1) is quantized: it can only take discrete values [91]. The number of "bound states" in this QCF depends on $V_{C} \cdot\left(2 R_{Q C P}\right)^{2}$ (see [91]): there is a range of values of $V_{C} \cdot\left(2 R_{Q C P}\right)^{2}$ for which there is only one energy level. Now, if we assume, for simplicity, that the QD size, $2 R_{Q C P}$, is so tiny that there is only one energy level, $E_{Q D}$, its associated wavefunction is a $1 s$-orbital $[6,93]$. We have solved the problem for a single, isolated QD with $R_{Q C P}=10 \mathrm{~nm}$ and $V_{C}=0.47 \mathrm{eV}$, typical in III-V semiconductors. There is only one bound state, whose energy is $E_{Q D}=0.4 \mathrm{eV}$. Its associated wave function $\psi_{Q D}$ is a spherical $1 s-$ orbital. We have represented its squared modulus, $\left|\psi_{Q D}\right|^{2}$, in both Cartesian (Figure $4 \mathrm{~b}$ ) and radial coordinates (Figure $4 \mathrm{c}$ ). The latter shows how $\left|\psi_{Q D}(r)\right|^{2}$ decreases very quickly as a function of the normalized radial coordinate, $r / R_{Q C P}$.

With this idea in mind, the potential in the complete system is a function that varies from one QD to another, taking the $-V_{C}$ value inside each QD and zero in the space among QDs:

$$
V \equiv V(\mathbf{r})=\left\{\begin{array}{cc}
-V_{C} & , \text { inside } a \text { QD at } \mathbf{r} \\
0 & \text {, outside } \mathrm{QDD},
\end{array}\right.
$$

where $\mathbf{r}$ is the position vector locating each QD.

\section{Modeling the QD System as a Spatial Network: The Proposed Model}

Aiming at generating the network associated with the proposed system, we represent any QD $i$ by means of a node, and we label this node using its ket $|i\rangle$, as shown in Figure 5a. Our next step is to generate the links in a way that makes physical sense according to QM, and also takes into account that the nodes are spatially embedded. As outlined in Section 1 when introducing our approach, we generate a link between two nodes (sites, kets), $|i\rangle$ and $|j\rangle$, located at $\mathbf{r}_{i}$ and $\mathbf{r}_{j}$, respectively, by computing to what extent their respective wave functions overlap. We compute the overlap between the wave functions $\psi_{Q D_{i}}$ and $\psi_{Q D_{j}}$ as the overlap integral [94] over all the physical space $S$

$$
\int_{S} \psi_{Q D_{j}}^{*} \psi_{Q D_{i}} d \mathbf{r}=\langle j \mid i\rangle=w_{i j}
$$

Note that, because of symmetry, $\langle j \mid i\rangle=\langle i \mid j\rangle$.

Using Expression (5), we have computed the overlap integral for nodes whose centers are separated by a normalized Euclidean distance $d_{E} / R_{Q C P}$. The result appears in Figure 6, where we have represented the overlap integral as a function of $d_{E} / R_{Q C P}$. We have highlighted two situations in Figure 6 for illustrative purposes. 

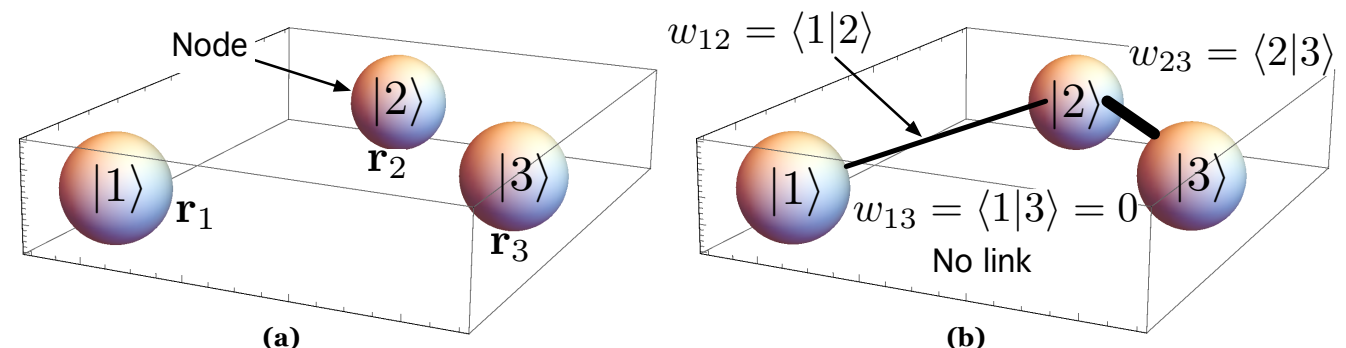

Figure 5. (a) System with three QDs. Vector $\mathbf{r}$ is the position vector in the metric space. We represent each QD as a node, and we label it with the ket notation $|i\rangle ;(\mathbf{b})$ methodology to form weighted links according to QMs. Each weight $w_{i j}$ is the overlap integral between $\psi_{Q D i}$ and $\psi_{Q D j}$ (or, equivalently, $\langle i \mid j\rangle$ in Dirac's notation).

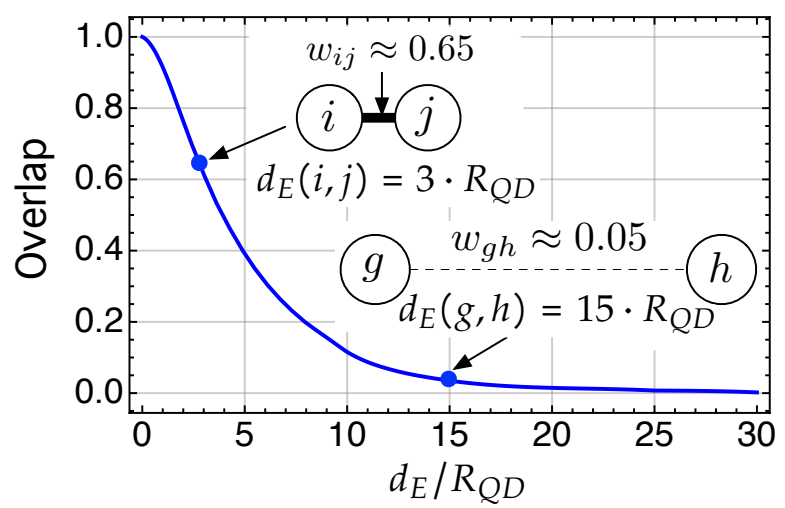

Figure 6. Overlap between the electron wave functions in two QDs as a function of the distance (between dot centers) normalized by the radius of the $Q D, d_{E} / R_{Q D}$. The insets aim to graphically illustrate how increasing the separation between the nodes implies a longer link with much less weight.

The first one corresponds to the inset in which the centers of the nodes (sites) $i$ and $j$ are separated by a distance $d_{E}(i, j)=3 \cdot R_{Q D}$ for which $\langle i \mid j\rangle=w_{i j} \approx 0.65$. Thus, we generate a link between nodes $i$ and $j$ whose weight is $w_{i j}=0.65$. The second inset corresponds to two nodes whose centers are at a $d_{E}(g, h)=15 \cdot R_{Q D}$ for which $w_{g h}=\langle g \mid h\rangle=w_{g f} \approx 0.05$. A link is generated, but has a very small weight $w_{g h} \approx 0.05$. Note that the overlap integral $w_{i j} \rightarrow 0$ when $d_{E}(i, j)>25 \cdot R_{Q D}$, and is $w_{i j}=0$ for $d_{E}(i, j) \geq 30 \cdot R_{Q D}$. That is, in our system, all those nodes whose centers are separated by a distance $d_{E} \geq 30 \cdot R_{Q D}=d_{E \text {, Lim }}$ (or limit distance) are not allowed to be linked. This is the case of nodes $|1\rangle$ and $|3\rangle$ in Figure 5, where $\langle 1 \mid 3\rangle=w_{13}=0$.

Although it does not seem clear yet at this point,

$$
d_{E, \operatorname{Lim}} \equiv d_{S},
$$

defines a new scale in the system, $d_{S}$. Note that our approach is a modified version of a RGG. As mentioned, an RGG is the simplest spatial network, consisting of randomly placing $N$ nodes in some metric space and connecting two nodes by a link if and only if their Euclidean distance is smaller than a given neighborhood radius, $r$. In our case, this is distance is $d_{E, \text { Lim }}$. The novelty of our approach is that any link between nodes $i$ and $j$ is characterized by a weight that involves the overlap integral between kets $|i\rangle$ and $|j\rangle$.

To advance in our model, it is necessary to introduce some essential concepts. The first one arises from the very interaction between nodes. When two nodes are directly connected by a link, they are then said to be "adjacent" or neighboring. The adjacency matrix A encodes the topology of a network, that is, whether or not there is a link $\left(a_{i j}=1\right.$ or $a_{i j}=0$ ) between any two pairs of nodes $i$ and $j$. Sometimes, this binary information 
encoding whether or not a node is connected to another is not enough, and it is necessary to quantify the "importance" of any link (the strength of a tie between two users in a social network, or the flow of electricity between two nodes in a power grid [35]) by assigning to each link a "weight". In that case, the matrix that encodes the connections is called weighted adjacency matrix W [41]. With our method, the weighted adjacency matrix corresponding to the network represented in Figure $5 b$ is

$$
\left(\begin{array}{ccc}
0 & \langle 1 \mid 2\rangle & \langle 1 \mid 3\rangle \\
\langle 2 \mid 1\rangle & 0 & \langle 2 \mid 3\rangle \\
\langle 3 \mid 1\rangle & \langle 3 \mid 2\rangle & 0
\end{array}\right)=\left(\begin{array}{ccc}
0 & \langle 1 \mid 2\rangle & 0 \\
\langle 2 \mid 1\rangle & 0 & \langle 2 \mid 3\rangle \\
0 & \langle 3 \mid 2\rangle & 0
\end{array}\right)
$$

which is symmetric (since $\langle i \mid j\rangle=\langle j \mid i\rangle$ in this quantum systems), off-diagonal and nonnegative. In particular, $\langle 1 \mid 3\rangle=\langle 3 \mid 1\rangle=0$ since there is no overlap between the electron wave functions in kets $|1\rangle$ and $|3\rangle$.

An interesting point in Expression (7) is that its matrix elements have in QM the meaning of probability amplitude, $\langle i \mid j\rangle$, which is related to the probability for an electron to be in $|i\rangle$ and $|j\rangle, \mathrm{P}_{i \sim j}$, as follows:

$$
\langle j \mid i\rangle^{*}\langle j \mid i\rangle=|\langle j \mid i\rangle|^{2} .
$$

We can now generalize the idea from the toy system in Figure 5 to the complete, complex, quantum system composed of $N$ QDs that are independently and uniformly distributed in the metric space $\mathbb{R}^{2}$. The corresponding adjacency matrix is thus an $N \times N$ weighted adjacency matrix $\mathbf{W}_{P A}$ whose matrix elements, $\langle i \mid j\rangle$, are the probability amplitude for an electron in kets $|i\rangle$ and $|j\rangle$,

$$
\left(\mathbf{W}_{P A}\right)_{i j}=\left\{\begin{array}{cc}
0 & , \text { if } i=i \\
\langle i \mid j\rangle & , \text { if } i \neq j
\end{array}\right.
$$

Once we have defined our weighted adjacency matrix, $\mathbf{W}_{P A}$ in (9) and interpreted its meaning in QM, we now have enough knowledge to represent the system as a network by using the undirected, weighted graph $\mathcal{G} \equiv \mathcal{G}\left(\mathcal{N}, \mathcal{L}, \mathbf{W}_{P A}\right)$, where $\mathcal{N}$ is the set of nodes $(\operatorname{card}(\mathcal{N})=N)$ and $\mathcal{L}$ is the set of links. We have specified the matrix $\mathbf{W}_{P A}$ in the triplet $\mathcal{G} \equiv \mathcal{G}\left(\mathcal{N}, \mathcal{L}, \mathbf{W}_{P A}\right)$ to emphasize the fact that the connections between the nodes are made using the $\mathbf{W}_{P A}$ matrix and not, for example, a conventional adjacency matrix $\mathbf{A}\left(a_{i j}=1\right.$ if $i$ and $j$ are directly linked; 0 otherwise), which would result in different results.

Note that, because of the way we have generated the links, the weighted adjacency matrix $\mathbf{W}_{P A}$ quantifies connections that have physical meaning according to QM, and explicitly includes the spatial structure of the system (remember Figure 6 and its associated, previous discussion). This is the key point that allows us to apply to $\mathbf{W}_{P A}$ techniques that are well known in network science. For instance, in addition to the weighted adjacency matrix, it is common to use the diagonal degree matrix $\mathbf{D}$, whose elements $D_{i}$ are the sum of weights of all links directly connecting node $i$ with others. In our particular system, D has physical meaning: using (9), $D_{i}=\sum_{i \neq j}\left(\mathbf{W}_{P A}\right)_{i}=\sum_{i \neq j}\langle i \mid j\rangle \equiv s_{A P_{i}}$ is the sum of the probability amplitudes on ket $|i\rangle$. We label it $s_{A P_{i}}$ to stress this physical meaning.

$\mathbf{W}_{P A}$ helps us obtain Laplacian matrices that will assist us in studying electron dynamics using CTQW, quantum walks that are continuous in time and discrete on space. See [64] for a very illustrative discussion on CTQW and their use in the simulation of quantum systems. The first type of Laplacian matrix, the (combinatorial) Laplacian, or simply, Laplacian matrix,

$$
\mathbf{L}=\mathbf{D}-\mathbf{W}_{P A} .
$$

Note that the Laplacian matrix $\mathbf{L}$ computed using the weighted adjacency matrix $\mathbf{W}_{P A}$ is different from the one used in other works $[72,84,95]$. In these approaches, the matrix elements of $\mathbf{L}$ are assumed to be equal $\gamma_{i j} \equiv \gamma=1$. In our approach, the matrix elements 
take different values since they depend on the involved overlap integrals (or probability amplitudes, $0 \leq w_{i j}<1$ ) and, as shown throughout the paper, they play a natural role in the probability for an electron to tunnel from one node to another. The Laplacian acts as a node to node transition matrix. The Hamiltonian of the CTQW can be written as $\mathbf{H}=\mathbf{L}[68,75,77,82,84,85,96-101]$.

The second one is the normalized Laplacian matrix [96], $\mathcal{L}_{N}=\mathbf{D}^{-1 / 2} \mathbf{L D}^{-1 / 2}$, an Hermitian operator that, according to the way we have defined $\mathbf{W}_{P A}$ in (9), has matrix elements in the form

$$
\left(\mathcal{L}_{N}\right)_{i j}=\left\{\begin{array}{cl}
1 & , \text { if } i=j \\
-\frac{\langle i \mid j\rangle}{\sqrt{S_{A P i}} \cdot \sqrt{S_{A P j}}} & , \text { if } i \neq j
\end{array}\right.
$$

$\mathcal{L}_{N}$ allows for generating the corresponding unitary CTQW [96] of an electron on our graph $\mathcal{G} \equiv \mathcal{G}\left(\mathcal{N}, \mathcal{L}, \mathbf{W}_{P A}\right)$ as

$$
\widehat{U}_{\mathcal{L}_{N}}(t)=e^{-i \mathcal{L}_{N} \cdot t}
$$

Note that, in the time evolution operator generated by $\mathcal{L}_{N}$ in (12), the imaginary unit makes $\widehat{U}_{\mathcal{L}_{N}}$ be unitary [75]. As in other $C N$ approaches $[74,76,102,103]$, we assume $\hbar \equiv 1$ so that time and energy can be treated as dimensionless. We will use $\widehat{U}_{\mathcal{L}_{N}}(t)$ to study the temporal evolution of our quantum system.

\section{Experimental Work: Simulations}

\subsection{Network Parameters}

The spatial constraints stated by the overlap integral have effects on $\mathrm{CN}$ parameters such as degree distribution, clustering, and average shortest path, defined as:

- The degree distribution of a network captures the probability $P(k)$ that a randomly chosen node exhibits "degree" $k$ (= number of links). $P(k)$ and its mean value $\langle k\rangle$ (mean degree) are very useful since it quantifies to what extent nodes are heterogeneous with respect to their connectivity. In fact, many real-world networks exhibit broad, heterogeneous degree distributions. In a degree-heterogeneous network, the probability to find a node with $k\rangle\langle k\rangle$ decreases slower than exponentially, leading to the existence of a non-negligible number of nodes with very high degrees. A key feature of such degree distributions is the so-called scale-free behavior [32], characterized by a degree distribution $P(k) \sim k^{-\gamma}$. This means that most of the nodes have very few links, while only a few nodes have a large percentage of all links. These most connected nodes are called "hubs".

- The clustering or transitivity [32] quantify the probability that two neighbors of a given node $i$ are connected. This concept is clear in social networks: the fact that usually "the friend of a friend is a friend" leads to high clustering coefficient. The "clustering coefficient" is a local property capturing "the density" of triangles in the graph, that is, two nodes that both are connected to a third node are also directly connected to each other. A node $i$ in the network has $k_{i}$ links that connects it to $k_{i}$ other nodes. The clustering coefficient of node $i$ is defined as the ratio between the number $M_{i}$ of links that actually exist between these $k_{i}$ nodes and the maximum possible number of links, that is, $C_{i}=2 M_{i} / k_{i}\left(k_{i}-1\right)$. The clustering coefficient of the whole network is:

$$
\langle\mathcal{C}\rangle=\frac{1}{N} \sum_{i} C_{i}
$$

- The average shortest path length, $\ell$, quantifies the extent to which a node is accessible from any other [32]. The average path length of a network is the average value of distances between any pair of nodes in the network:

$$
\ell=\frac{1}{N(N-1)} \sum_{i \neq j} d_{i j}
$$


where $d_{i j}$ is the distance between node $i$ and node $j$. The length of the shortest path between two nodes $i$ and $j$ in a network is the minimum number of links for going from node $i$ to $j$. Its average is computed over all possible pairs of nodes. When $\ell$ is small when compared to the "network size" (number of nodes, $N$ ), the small-world property arises. Intuitively, this means that any pair of nodes are relatively "close". Mathematically, this means that the average shortest path scales logarithmically with the network size [32]: $\ell \sim \ln N$.

The fact that an electron cannot tunnel from one QD to another that is at a distance longer than $d_{E, L i m}$ leads to inhibiting the existence of long-range links to connect hubs (that is, shortcuts are not allowed). The increasing tendency to establish connections in the neighborhood $\left(d<d_{E, \text { Lim }}\right)$ leads to a high clustering coefficient $(\langle\mathcal{C}\rangle \approx 0.57)$.

\subsection{The Network Has a Percolation Transition as the Dot Density Increases}

Consider the density of QDs as $\rho_{Q D}=N / A$, where $A=1$ is the area of a unit square. When $\rho_{Q D}$ is very small (and, so does $\langle k\rangle, k$ being the number of links per node), the nodes are so far apart that there is no overlap between the wave functions and, as a consequence, there is no links: $\langle k\rangle \rightarrow 0$ and there are $N$ isolated nodes.

As $\rho_{Q D}$ increases (and, so does $\langle k\rangle$ ), the QDs are closer and closer and electron wavefunctions in some of them start to overlap, causing the formation of links among some of them. As a consequence, small clusters begin to appear. They are disconnected from each other. These isolated subnetworks are called components and all of them have similar size.

However, there is a value of $\langle k\rangle$ for which one of the clusters becomes dominant and begins to grow to the detriment of the others. This cluster is called a giant component (GC). The fraction or normalized size of the giant component with respect to the total number of nodes is quantified as $S_{G C}=N_{G C} / N$. Figure 7 shows $S_{G C}$ of our network as a function of the average node degree $\langle k\rangle$. Note that, for $\langle k\rangle\langle 3.4$, there is no giant component. There are several disconnected networks, many of then being trees. The point $\langle k\rangle=3.4$ in Figure 7 seems to be a critical point at which $S_{G C}$ has an abrupt transition. For $3.4<\langle k\rangle<10$, there is a single giant component with small clusters. For $\langle k\rangle \geq 10$, there is only a single giant component, that is, the network is connected.

This formation of a GC on a macroscopic scale is an example of percolation transition [104]. The fraction of nodes belonging to the giant cluster becomes the order parameter of the percolation transition. Following [104], we have denoted it as $m(\langle k\rangle)$. Ref. [104] explores the most recent advances of percolation theory in $\mathrm{CN}$, and studies the order parameter $m$ for continuous, explosive, discontinuous, and hybrid percolation transitions. According to [104], our network exhibits a Hybrid Percolation Transition (HPT) since it has properties of both second-order (critical phenomena) and first-order (abrupt jump of the order parameter) phase transitions at the same transition point, $\langle k\rangle_{C}$, in our case.

In our case, $m(\langle k\rangle) \equiv S_{G C}(\langle k\rangle)$ fulfills

$$
m(\langle k\rangle)=\left\{\begin{array}{cl}
0 & , \text { if }\langle k\rangle<\langle k\rangle_{C} \\
m_{0}+r \cdot\left(\langle k\rangle-\langle k\rangle_{C}\right)^{\beta_{m}} & , \text { if }\langle k\rangle \geq\langle k\rangle_{C}
\end{array}\right.
$$

where $m_{0}$ and $r$ are constants, and $\beta_{m}$ is the critical exponent of the order parameter. For $\langle k\rangle_{C} \geq 3.4, m(\langle k\rangle)=0.0492937+0.788699(\langle k\rangle-3.4)^{0.099}$. 


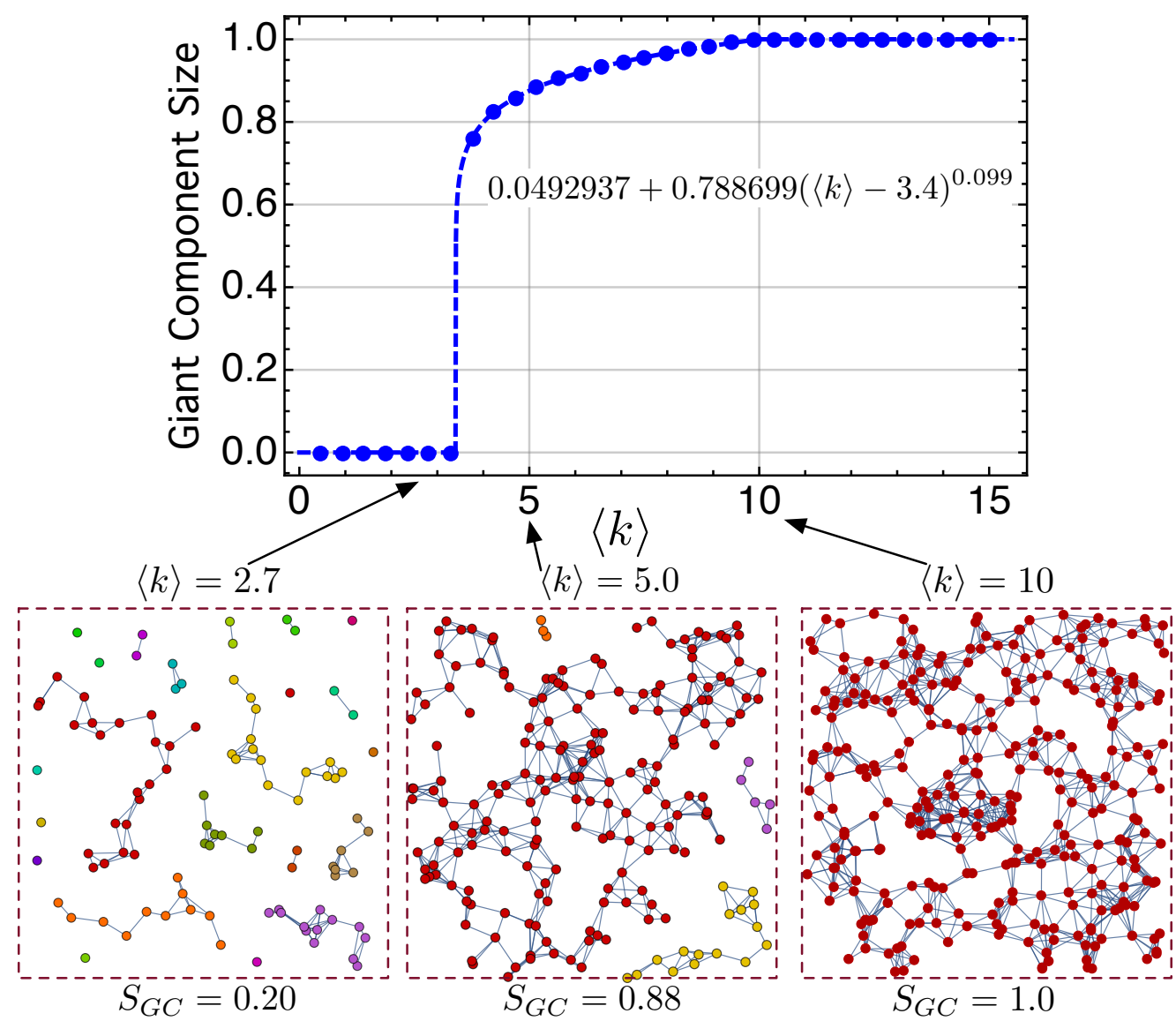

Figure 7. Normalized size of the giant component with respect to the total number of nodes, $S_{G C}=N_{G C} / N$, as a function of the average node degree $\langle k\rangle$. See the main text for further details.

\subsection{Studying the Emergence of Electron Transport}

We characterize the network's transport efficiency by using the average return probability $\bar{\alpha}(t)$, defined as [85]

$$
\bar{\alpha}(t)=\frac{1}{N} \sum_{j=1}^{N}\left|\left\langle j\left|\widehat{U}, \mathcal{L}_{N}(t)\right| j\right\rangle\right|^{2},
$$

where the operator $\widehat{U}_{\mathcal{L}_{N}}(t)$, stated in Equation (12), is the unitary time evolution operator governing the evolution of the probability amplitudes. Please note that, as shown in a number of papers $[68,75,77,82,84,85,96-101]$, the Hamiltonian of the network is the Laplacian matrix (also called Connectivity matrix in some contexts).

High values of $\bar{\alpha}(t)$ suggest inefficient transport since the quantum particle tends to remain at the initial node [85]. On the contrary, $\bar{\alpha}(t) \ll 1$ means that the electron, localized at the initial node in $t=0$, tends to be delocalized, with different probability components on each node.

With this concepts in mind, we define the quantum transport efficiency as

$$
\eta_{Q T}(t)=1-\bar{\alpha}(t) .
$$

Figure 8 shows the quantum transport efficiency $\eta_{Q T}$ stated by (17) as a function of the average node degree $\langle k\rangle, k$ being the number of links per node. Note that $\eta_{Q T}=0$ for $\langle k\rangle<10$ while there is and abrupt transition at $\langle k\rangle=10$ so that $\eta_{Q T} \approx 1$ for $\langle k\rangle>10$. 


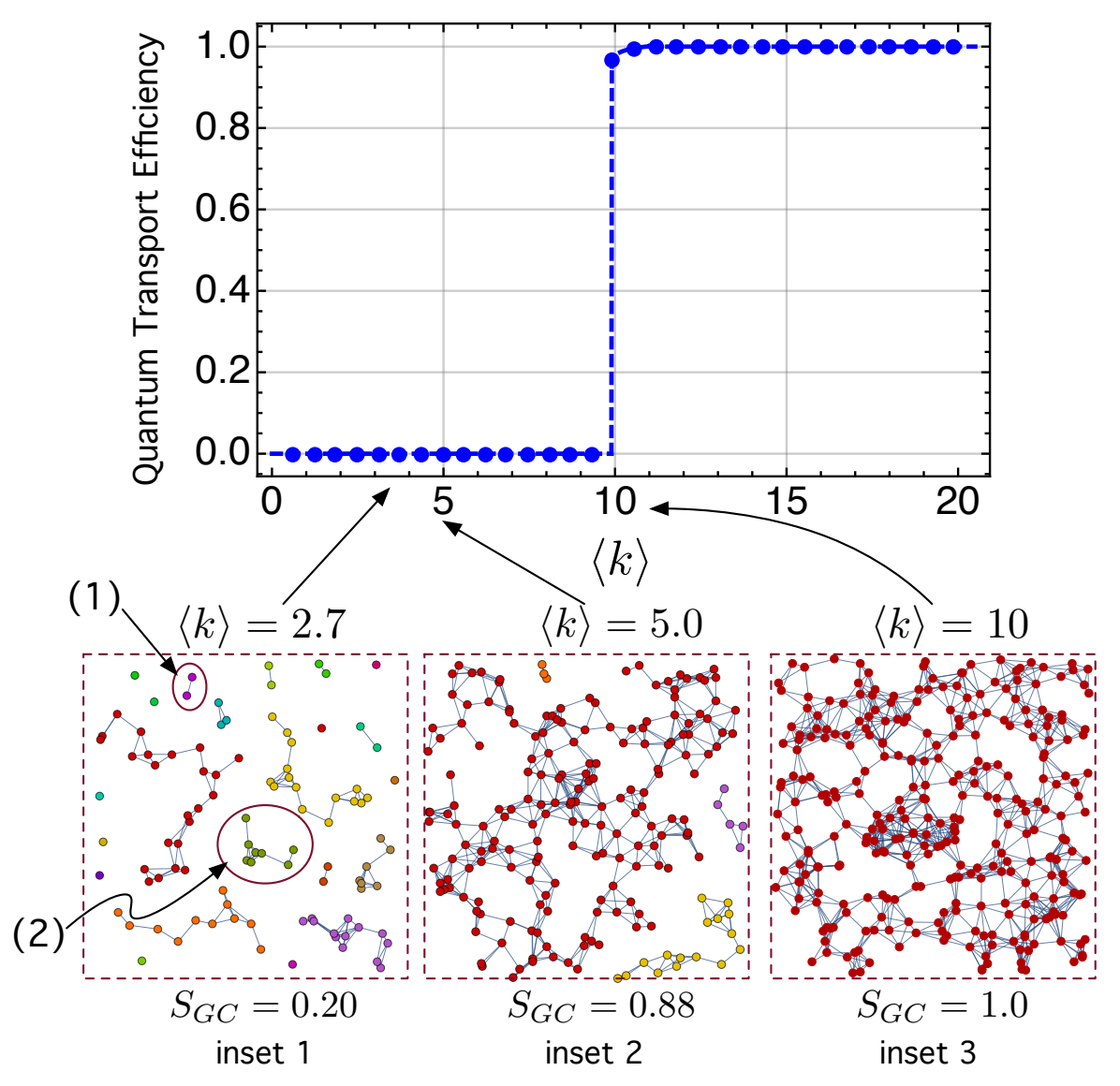

Figure 8. Quantum transport efficiency as a function of $\langle k\rangle$. Insets " 1 ", " 2 " and " 3 " shows network connectivity at different values of $\langle k\rangle . S_{G C}=N_{G C} / N$ is the fraction (normalized size) of the giant component.

The results shown in Figure 8 has been computed with $t=500$. Note that time and energy can be treated as dimensionless when assuming $\hbar \equiv 1$, as mentioned before $[74,76,102,103]$.

To explain this result, we have studied what happens to the connectivity of the network when the density of QDs per unit area $(A), \rho_{Q D}=N / A$, increases. When $\rho_{Q D}$ is very small (and, so does $\langle k\rangle$ ), the nodes are so far apart that there is no overlap between wavefunctions and, as a consequence, there is no links: $\langle k\rangle \rightarrow 0$, and there are $N$ isolated nodes. As $\rho_{Q D}$ increases (and so does $\langle k\rangle$ ), the QDs are closer and closer and the electron wavefunctions in some of them start to overlap, causing the formation of links among some of them. This is the case of the two nodes labeled "(1)" in Figure 8. Small clusters begin to appear too (cluster labeled "(2)"). Note that they are disconnected from each other. These isolated subnetworks are called components and many of them have a similar size. In any component, the electron can tunnel among the involved QDs. This is just the situation in the below, leftmost inset, called "inset 1".

However, as $\langle k\rangle$ continues to grow, one of the clusters becomes dominant and begins to grow more and more as forming links to other smaller clusters. This cluster is called a giant component (GC). The fraction or normalized size of the giant component with respect to the total number of nodes is quantified as $S_{G C}=N_{G C} / N$. In "inset 2", with $\langle k\rangle=5$, we can see that there are small clusters along with a single GC whose size is $S_{G C}=0.88$.

Just when $\langle k\rangle$ increases up to $\langle k\rangle=10$, one single GC $\left(S_{G C}=1\right)$ appears, which has captured all the other small components, making the complete network be connected. The electron can thus tunnel from any node to any other node. When the network is connected, an electron always has at least one path to pass from any node $j$ to another. As a consequence, $\bar{\alpha}(t)=0$ in Equation (16) since the electron is no longer confined at node 
$j$, and can now behave as an extended wave function whose components are distributed (although with different probability components) between different nodes (see Figure 9). As $\bar{\alpha}(t) \approx 0$, then the quantum transport efficiency (17) is $\eta_{Q T}(t)=1-\bar{\alpha}(t) \approx 1$.

Figure 9 is an example of the electron probability components, $|\langle n \mid \psi\rangle|^{2}$, on each of the kets $|n\rangle$ of a network with $N=100$ nodes. In this example, the electron was localized in node $|7\rangle$ at $t=0$ (initial state), and after applying the unitary evolution operator (12) for $t=500$, it has evolved to a extended wave function whose probability components $|\langle n \mid \psi\rangle|^{2}$ are distributed over the $N=100$ nodes.

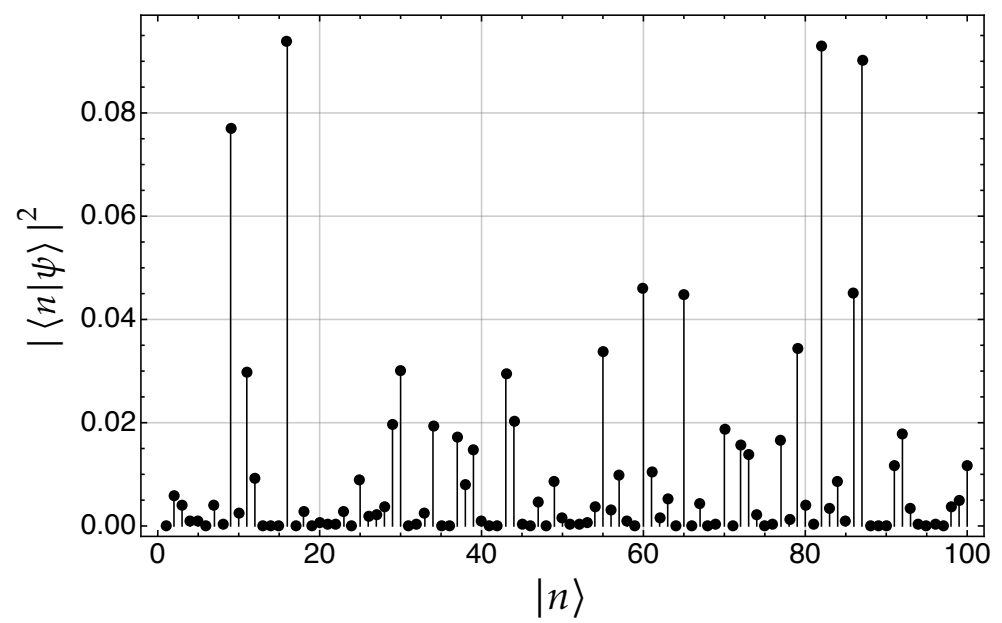

Figure 9. Electron probability components, $|\langle n \mid \psi\rangle|^{2}$, on each of the kets $|n\rangle$ of a connected network with $N=100$ nodes.

For the sake of clarity, Table 1 lists the corresponding numerical values represented in Figure 9. Please note that the sum of the probability components in the whole system is $\sum_{n=1}^{N=100}|\langle n \mid \psi\rangle|^{2}=1$.

Table 1. List of the electron probability components, $|\langle n \mid \psi\rangle|^{2}$, on each of the kets $|n\rangle$ of a connected network with $N=100$ nodes. The sum of the probability components in the whole system is $\sum_{n=1}^{N=100}|\langle n \mid \psi\rangle|^{2}=1$.

\begin{tabular}{ccccc}
0.000172176 & 0.00582029 & 0.00387988 & 0.00106017 & 0.000970012 \\
0.0000719148 & 0.00413783 & 0.000454207 & 0.0768675 & 0.00242522 \\
0.0298138 & 0.00921407 & 0.0000565646 & 0.000102786 & 0.0000761972 \\
0.0938865 & 0.00010824 & 0.0027705 & 0.0000545876 & 0.000735455 \\
0.000247052 & 0.000190905 & 0.00274959 & 0.0000908889 & 0.00886395 \\
0.00197876 & 0.00202883 & 0.003812 & 0.0197223 & 0.0300932 \\
0.000148517 & 0.000360844 & 0.00247567 & 0.0193588 & 0.0000203756 \\
0.0000281 & 0.0172012 & 0.00814919 & 0.0147341 & 0.00103512 \\
0.000150275 & 0.0000862307 & 0.0294795 & 0.0203633 & 0.00025177 \\
0.0000518012 & 0.00469129 & 0.0000315912 & 0.00847614 & 0.00144027 \\
0.000285581 & 0.000261477 & 0.000582733 & 0.00371945 & 0.0336232 \\
0.0032206 & 0.00980407 & 0.000807111 & 0.000121867 & 0.0459497 \\
0.0105552 & 0.00168941 & 0.00534086 & 0.000115002 & 0.0447992 \\
0.0001218 & 0.00431284 & $8.72451 \times 10-6$ & 0.000201637 & 0.0188286 \\
0.000133976 & 0.0156714 & 0.0137177 & 0.0022036 & 0.000168711 \\
0.000250133 & 0.0166772 & 0.00114926 & 0.0344186 & 0.00393284 \\
0.000493777 & 0.0928017 & 0.00341317 & 0.00876622 & 0.000893781 \\
0.0451846 & 0.0901622 & 0.0000291204 & 0.0000476467 & 0.0000574528 \\
0.0117769 & 0.0178166 & 0.0034605 & 0.000244178 & 0.000142244 \\
0.00037752 & 0.000129045 & 0.00373535 & 0.00499453 & 0.0118115 \\
\hline
\end{tabular}




\section{Potential Applications, Strengths, and Weaknesses of the Proposed Method}

\subsection{Prospective Applications}

To the best of our knowledge, there are two possible groups of applications: intermediate band (IB) materials and light-harvesting materials.

\subsubsection{Intermediate Band Materials}

The first potential application of the proposed method could be found within the field of IB materials to put into practice the concept of intermediate band solar cell (IBSC) [105]. This solar cell has an isolated IB within what, otherwise, would be the semiconductor gap, $E_{G}$. The IBSC was proposed by Luque and Martí in [106] and exhibits a limiting efficiency of 63.2\%, much higher than the Shockley-Queisser (SQ) limit [107] of the singlegap solar cell (40.7\%). Unlike other sub-bandgap absorbing proposals, the IBSC concept surpasses the SQ limit by means of: (1) increasing the photogenerated current via the two-step absorption of sub-bangap photons via an IB located within the gap $E_{G}$; and (2) without degrading the cell output voltage. Several technological approaches have been proposed aiming to obtain IB materials. The first one, the QD approach has led to the quantum dot intermediate band solar cell (QD-IBSC) [108], the first device on which it has been possible to experimentally prove the two concepts the IBSC is based on [109-112]. A sufficiently dense array of QDs with a single bound state leads to an IB material. Regarding this, a possible future application of our method could consist in exploring the electron conductivity in the IB that arises from the bound states within the QDs. A second feasible strategy for obtaining IB materials is based on semiconductor bulk materials containing a high density of adequate deep-level impurities and those corresponding to materials that "naturally" have an IB (theoretically predicted by ab-initio methods) [113-119]. Regarding the use of deep-level impurities, the proposed method could potentially be used to study when a sufficiently large density of impurities produces a transition from localized states (in the impurities) to states that are extended to the whole volume of the host semiconductor. It could be interesting to compare with experimental data [120,121] and theoretical models [122]. Putting it simply, deep centers transform into a band when their density is sufficiently high (Mott transition) [123,124], and, as a consequence, the electron wave-function becomes extended instead of localized. Regarding this, the experimental test of the theoretical model stated in [122] have been recently proved in silicon implanted with Ti [120] and in silicon supersaturated with sulfur [121].

\subsubsection{Light-Harvesting Materials}

In the field of light-harvesting materials [125], QDs have been found to be promising light-harvesting materials because of their size-, shape-, and composition-dependent electronic properties, and exciton generation after photoexcitation [126]. The key challenge is to model and design nanoscale materials with tailored properties for light harvesting work. Regarding this, our proposal could be used to explore the transport of energy (excitons) in light-harvesting QDs [126], light-harvesting molecules [125,127,128], polymer nanoparticles [129], dimers, and networks [130].

\subsection{Strengths and Weaknesses}

The proposed method has a set of strengths and weaknesses. Among the strengths, the method allows for obtaining more realistic simulations of nanostructures than others found in the literature, where the fact that the tunneling probability decreases exponentially with the distance is not considered. The method is also generalizable to nanostructures other than QDs, such as impurities in semiconductors, light-harvesting molecules, etc. Another advantage is that it allows obtaining an approximation of the electron behavior in systems with a high number of components per area unit (whether they are QDs or impurities). The main weakness of the proposed method is that it is a first approach in the sense that identical quantum dots have been assumed. However, self-assembled quantum dot growth 
methods produce ensembles of quantum dots that have size dispersion, which leads to a dispersion in bound levels from one QD to another. The probability of electron hopping from a QD to another with the same energy level must be considered when computing the weight of links. Additionally, it is necessary to consider not only the electron system but also the phonon system, which provides/absorbs the energy when an electron hops from one bound state in a QD to another with different energy. Because of its complexity, we leave this refinement for future work.

\section{Summary and Conclusions}

This paper has proposed the use of a special class of Random Geometric Graphs (RGG) to model systems formed by $N$ disordered quantum dots (QDs). While discerning what a node is seems easy ( $Q D \equiv$ node), what requires a bit more care and physical intuition is determining how the links between QDs are formed in such a way that they have meaning. Specifically, in the network model that we have proposed, the most novel aspect is the link formation mechanism between nodes (三QDs): any link between two nodes $i$ and $j$ is formed if and only if the corresponding electron wave function at such nodes have non-zero overlap. Any link has thus a weight $w_{i j}$ that is the real number $\left(0 \leq w_{i j}<1\right)$ corresponding to the electron overlap integral (also called probability amplitude (PA)).

The aforementioned link formation mechanism leads to a $N \times N$ weighted adjacency matrix $\mathbf{W}_{P A}$ whose matrix elements are the probability amplitudes for a single electron in the involved nodes. The corresponding Laplacian matrix $\mathbf{L}$, which assists in computing continuous time quantum walks (CTQW) on the associated network, is different from the one used in other works $[72,84,95]$. In these approaches, the matrix elements of $\mathbf{L}$ are assumed to be equal $\gamma_{i j} \equiv \gamma=1$. In our approach, the matrix elements take different values since they depend on the involved overlap integrals (or probability amplitudes, $0 \leq w_{i j}<1$ ) and, as shown throughout the paper, they play a natural role in the probability for an electron to tunnel from one node to another.

Regarding this, our main results point out:

1. The spatial network generated by the proposed model prohibits the existence of shortcuts between distant nodes because of the impossibility of the electron tunneling between two very distant QDs. This leads, as expected, to high clustering coefficient and makes it impossible for the network to be small-world.

2. The proposed network is also able to capture the inner properties of the QD system: it predicts the system quantum state, its time evolution, and the emergence of quantum transport (QT) as the mean node degree increases (or, equivalently, when the QD increases). In fact, QT efficiency exhibits an abrupt change, from electron localization (no QT) to delocalization (QT emerges), which has also been observed in [60], although with a different approach.

Author Contributions: L.C. has worked on the conceptualization, methodology, software, investigation, validation, writing - original draft preparation and writing-review and editing. J.C.N.-B. has worked on the conceptualization, methodology, and writing-review and editing. All authors have read and agreed to the published version of the manuscript.

Funding: This research has been partially funded by the Ministerio de Economía y Competitividad of the Spanish Government (Grant No. TIN2017-85887-C2-2-P).

Conflicts of Interest: The authors declare no conflict of interest. 


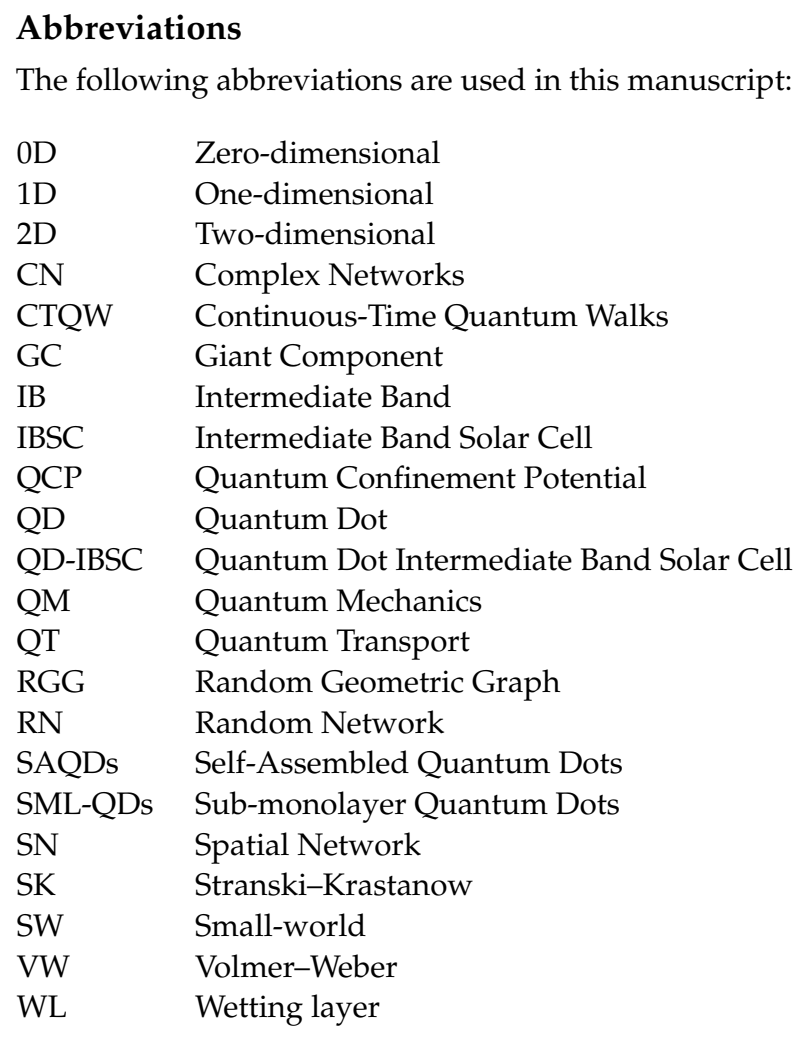

\section{Appendix A}

A Adjacency matrix of a graph $\mathcal{G}$.

$a_{i j} \quad$ Element of the adjacency matrix $\mathbf{A}$

$\bar{\alpha}(t) \quad$ Average return probability

$\langle\mathcal{C}\rangle \quad$ Mean clustering coefficient of a network.

D Node degree matrix: $\operatorname{diag}\left(k_{1}, \cdots, k_{N}\right)$. It is the diagonal matrix formed from the nodes degrees.

$d_{E}(i, j) \quad$ Euclidean distance between any pair of nodes $i$ and $j$ in a network.

$d_{i j} \quad$ Distance between two nodes $i$ and $j$. It is the length of the shortest path (geodesic path) between them, that is, the minimum number of links when going from one node to the other.

$d_{E, \text { Lim }} \quad d_{E, \text { Lim }} \equiv d_{S}$ Euclidean distance limit beyond which there is no link formation.

$E_{Q D} \quad$ Discrete electron energy in a quantum dot (QD).

$\eta_{Q T} \quad$ Quantum transport efficiency.

$\mathcal{G} \quad$ Graph $\mathcal{G} \equiv \mathcal{G}\left(\mathcal{N}, \mathcal{L}, \mathbf{W}_{P A}\right)$, where $\mathcal{N}$ is the set of nodes $(\operatorname{card}(\mathcal{N})=N), \mathcal{L}$ is the set of links, and $\mathbf{W}_{P A}$ is weighted adjacency matrix that emerges from our method to link formation.

$\widehat{H} \quad$ Hamiltonian operator corresponding to the total energy of a quantum system.

H Hamiltonian in matrix form.

h Planck constant.

$\hbar \quad$ Reduced Planck constant.

|i) Ket vector in the Hilbert space $\mathcal{H}$. It corresponds to the electron wave function in nanostructure ( $\equiv$ site $\equiv$ node $\equiv$ ket) $i$.

$\langle i| \quad$ Bra vector in the dual space corresponding to the ket $|i\rangle \in \mathcal{H}$

$\langle k\rangle \quad$ Average node degree.

$k_{i} \quad$ Degree of a node $i$. It is the number of links connecting $i$ to any other node.

$\ell \quad$ Average path length of a network. It is the mean value of distances between any pair of nodes in the network.

$\mathcal{L} \quad$ Set of links (edges) of a network (graph).

L Laplacian matrix of a graph $\mathcal{G}$. 


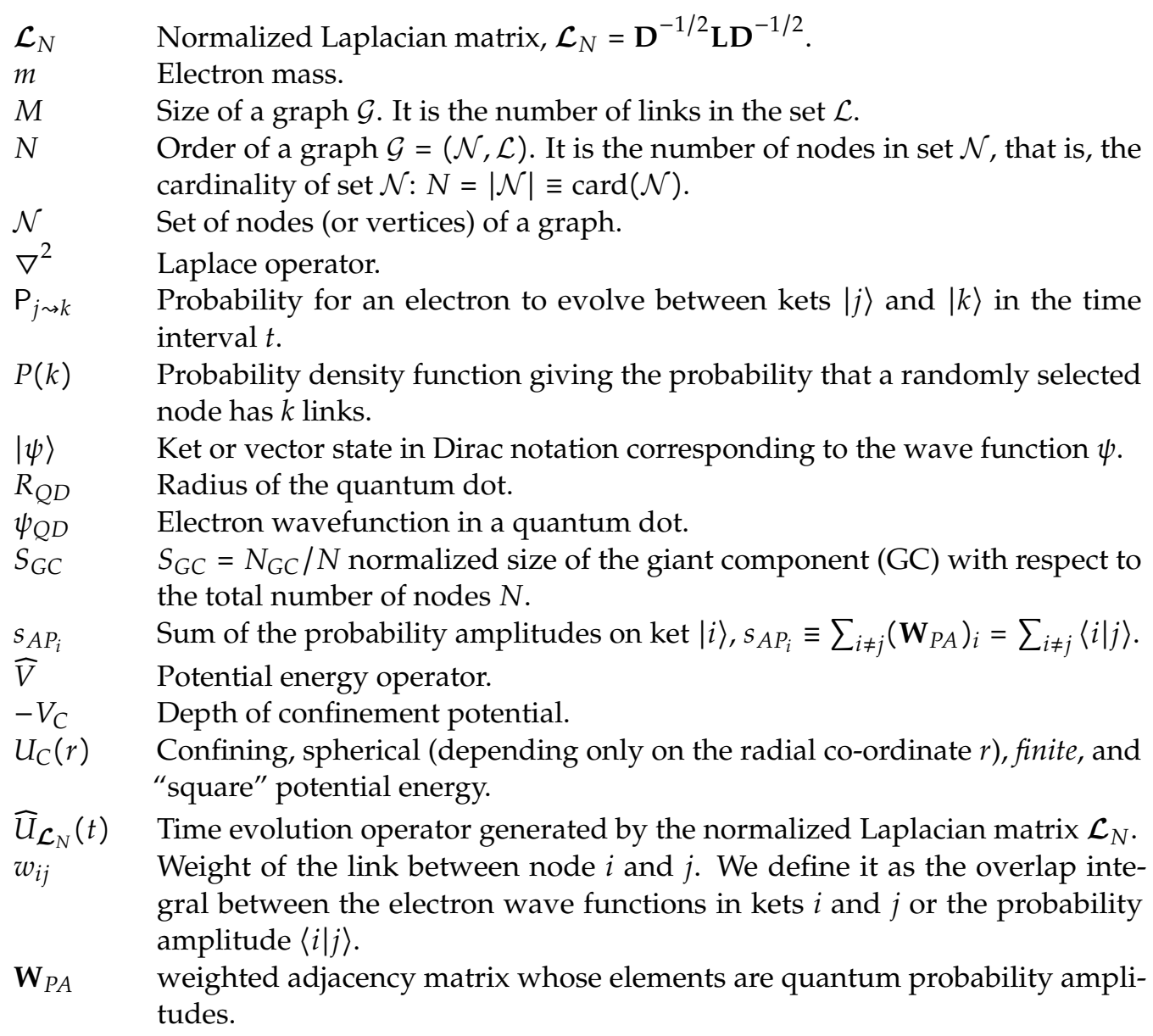

\section{References}

1. Cotta, M.A. Quantum Dots and Their Applications: What Lies Ahead? ACS Appl. Nano Mater. 2020, 3, 4920-4924. [CrossRef]

2. Harrison, P.; Valavanis, A. Quantum Wells, Wires and Dots: Theoretical and Computational Physics of Semiconductor Nanostructures; John Wiley \& Sons: Hoboken, NJ, USA, 2016.

3. Sengupta, S.; Chakrabarti, S. Structural, Optical and Spectral Behaviour of InAs-based Quantum Dot Heterostructures: Applications for High-performance Infrared Photodetectors; Springer: Berlin/Heidelberg, Germany, 2017.

4. Nowozin, T. Self-Organized Quantum Dots for Memories: Electronic Properties and Carrier Dynamics; Springer Science \& Business Media: Berlin/Heidelberg, Germany, 2013.

5. Cipriano, L.A.; Di Liberto, G.; Tosoni, S.; Pacchioni, G. Quantum confinement in group III-V semiconductor 2D nanostructures. Nanoscale 2020, 12, 17494-17501. [CrossRef]

6. Bimberg, D.; Grundmann, M.; Ledentsov, N.N. Quantum Dot Heterostructures; John Wiley \& Sons: Hoboken, NJ, USA, 1999.

7. Wang, Z.M. Self-Assembled Quantum Dots; Springer Science \& Business Media: Berlin/Heidelberg, Germany, $2007 ;$ Volume 1.

8. Kim, J.S.; Shin, J.C.; Kim, J.O.; Noh, S.K.; Lee, S.J.; Krishna, S. Photoluminescence study of InAs/InGaAs sub-monolayer quantum dot infrared photodetectors with various numbers of multiple stack layers. J. Lumin. 2019, 207, 512-519.

9. Leon, R.; Lobo, C.; Chin, T.; Woodall, J.; Fafard, S.; Ruvimov, S.; Liliental-Weber, Z.; Stevens Kalceff, M. Self-forming InAs/GaP quantum dots by direct island growth. Appl. Phys. Lett. 1998, 72, 1356-1358. [CrossRef]

10. Germann, T.; Strittmatter, A.; Pohl, J.; Pohl, U.; Bimberg, D.; Rautiainen, J.; Guina, M.; Okhotnikov, O. High-power semiconductor disk laser based on In As/ Ga As submonolayer quantum dots. Appl. Phys. Lett. 2008, 92, 101123. [CrossRef]

11. Xu, Z.; Birkedal, D.; Hvam, J.M.; Zhao, Z.; Liu, Y.; Yang, K.; Kanjilal, A.; Sadowski, J. Structure and optical anisotropy of vertically correlated submonolayer InAs/GaAs quantum dots. Appl. Phys. Lett. 2003, 82, 3859-3861. [CrossRef]

12. Kiyama, H.; Korsch, A.; Nagai, N.; Kanai, Y.; Matsumoto, K.; Hirakawa, K.; Oiwa, A. Single-electron charge sensing in self-assembled quantum dots. Sci. Rep. 2018, 8, 1-6. [CrossRef]

13. Gao, W.; Fallahi, P.; Togan, E.; Miguel-Sánchez, J.; Imamoglu, A. Observation of entanglement between a quantum dot spin and a single photon. Nature 2012, 491, 426-430. [CrossRef]

14. De Greve, K.; Yu, L.; McMahon, P.L.; Pelc, J.S.; Natarajan, C.M.; Kim, N.Y.; Abe, E.; Maier, S.; Schneider, C.; Kamp, M.; et al. Quantum-dot spin-photon entanglement via frequency downconversion to telecom wavelength. Nature 2012, 491, 421-425. [CrossRef] 
15. Michler, P.; Kiraz, A.; Becher, C.; Schoenfeld, W.; Petroff, P.; Zhang, L.; Hu, E.; Imamoglu, A. A quantum dot single-photon turnstile device. Science 2000, 290, 2282-2285. [CrossRef]

16. Press, D.; Ladd, T.D.; Zhang, B.; Yamamoto, Y. Complete quantum control of a single quantum dot spin using ultrafast optical pulses. Nature 2008, 456, 218-221. [CrossRef] [PubMed]

17. Deacon, R.S.; Oiwa, A.; Sailer, J.; Baba, S.; Kanai, Y.; Shibata, K.; Hirakawa, K.; Tarucha, S. Cooper pair splitting in parallel quantum dot Josephson junctions. Nat. Commun. 2015, 6, 1-7.

18. Hamaya, K.; Masubuchi, S.; Kawamura, M.; Machida, T.; Jung, M.; Shibata, K.; Hirakawa, K.; Taniyama, T.; Ishida, S.; Arakawa, Y. Spin transport through a single self-assembled InAs quantum dot with ferromagnetic leads. Appl. Phys. Lett. 2007, 90, 053108. [CrossRef]

19. Kanai, Y.; Deacon, R.; Takahashi, S.; Oiwa, A.; Yoshida, K.; Shibata, K.; Hirakawa, K.; Tokura, Y.; Tarucha, S. Electrically tuned spin-orbit interaction in an InAs self-assembled quantum dot. Nat. Nanotechnol. 2011, 6, 511-516. [CrossRef] [PubMed]

20. Takahashi, S.; Deacon, R.; Oiwa, A.; Shibata, K.; Hirakawa, K.; Tarucha, S. Electrically tunable three-dimensional g-factor anisotropy in single InAs self-assembled quantum dots. Phys. Rev. B 2013, 87, 161302. [CrossRef]

21. Kanai, Y.; Deacon, R.; Oiwa, A.; Yoshida, K.; Shibata, K.; Hirakawa, K.; Tarucha, S. Electrical control of Kondo effect and superconducting transport in a side-gated InAs quantum dot Josephson junction. Phys. Rev. B 2010, 82, 054512. [CrossRef]

22. Qi, H.; Wang, S.; Jiang, X.; Fang, Y.; Wang, A.; Shen, H.; Du, Z. Research progress and challenges of blue light-emitting diodes based on II-VI semiconductor quantum dots. J. Mater. Chem. C 2020, 8, 10160-10173. [CrossRef]

23. Lv, Z.; Wang, Y.; Chen, J.; Wang, J.; Zhou, Y.; Han, S.T. Semiconductor quantum dots for memories and neuromorphic computing systems. Chem. Rev. 2020, 120, 3941-4006. [CrossRef]

24. Chen, J.; Du, W.; Shi, J.; Li, M.; Wang, Y.; Zhang, Q.; Liu, X. Perovskite quantum dot lasers. InfoMat 2020, 2, 170-183. [CrossRef]

25. Geiregat, P.; Van Thourhout, D.; Hens, Z. A bright future for colloidal quantum dot lasers. NPG Asia Mater. 2019, 11, 1-8. [CrossRef]

26. Norman, J.C.; Jung, D.; Zhang, Z.; Wan, Y.; Liu, S.; Shang, C.; Herrick, R.W.; Chow, W.W.; Gossard, A.C.; Bowers, J.E. A review of high-performance quantum dot lasers on silicon. IEEE J. Quantum Electron. 2019, 55, 1-11. [CrossRef]

27. Bimberg, D.; Kirstaedter, N.; Ledentsov, N.; Alferov, Z.I.; Kop'Ev, P.; Ustinov, V. InGaAs-GaAs quantum-dot lasers. IEEE J. Sel. Top. Quantum Electron. 1997, 3, 196-205. [CrossRef]

28. Vichi, S.; Bietti, S.; Khalili, A.; Costanzo, M.; Cappelluti, F.; Esposito, L.; Somaschini, C.; Fedorov, A.; Tsukamoto, S.; Rauter, P.; et al. Droplet epitaxy quantum dot based infrared photodetectors. Nanotechnology 2020, 31, 245203. [CrossRef] [PubMed]

29. Ren, A.; Yuan, L.; Xu, H.; Wu, J.; Wang, Z. Recent progress of III-V quantum dot infrared photodetectors on silicon. J. Mater. Chem. C 2019, 7, 14441-14453. [CrossRef]

30. Sogabe, T.; Shen, Q.; Yamaguchi, K. Recent progress on quantum dot solar cells: A review. J. Photonics Energy $2016,6,040901$. [CrossRef]

31. Grundmann, M.; Stier, O.; Bimberg, D. InAs/GaAs pyramidal quantum dots: Strain distribution, optical phonons, and electronic structure. Phys. Rev. B 1995, 52, 11969. [CrossRef]

32. Barabási, A.L. Network Science; Cambridge University Press: Cambridge, UK, 2016.

33. Boccaletti, S.; Latora, V.; Moreno, Y.; Chavez, M.; Hwang, D.U. Complex networks: Structure and dynamics. Phys. Rep. 2006, 424, 175-308. [CrossRef]

34. Strogatz, S.H. Exploring complex networks. Nature 2001, 410, 268-276. [CrossRef]

35. Cuadra, L.; Salcedo-Sanz, S.; Del Ser, J.; Jiménez-Fernández, S.; Geem, Z.W. A critical review of robustness in power grids using complex networks concepts. Energies 2015, 8, 9211-9265. [CrossRef]

36. Cuadra, L.; Pino, M.D.; Nieto-Borge, J.C.; Salcedo-Sanz, S. Optimizing the structure of distribution smart grids with renewable generation against abnormal conditions: A complex networks approach with evolutionary algorithms. Energies 2017, 10, 1097. [CrossRef]

37. Doyle, J.C.; Alderson, D.L.; Li, L.; Low, S.; Roughan, M.; Shalunov, S.; Tanaka, R.; Willinger, W. The "robust yet fragile" nature of the Internet. Proc. Natl. Acad. Sci. USA 2005, 102, 14497-14502. [CrossRef] [PubMed]

38. Chimal-Eguía, J.C.; Castillo-Montiel, E.; Paez-Hernández, R.T. Properties of the vascular networks in malignant tumors. Entropy 2020, 22, 166. [CrossRef] [PubMed]

39. Braun, P.; Gingras, A.C. History of protein-protein interactions: From egg-white to complex networks. Proteomics 2012, 12, 1478-1498. [CrossRef] [PubMed]

40. Guimera, R.; Amaral, L.A.N. Functional cartography of complex metabolic networks. Nature 2005, 433, 895-900. [CrossRef] [PubMed]

41. Newman, M. Networks; Oxford University Press: Oxford, UK, 2018.

42. Albert, R.; Barabási, A.L. Statistical mechanics of complex networks. Rev. Mod. Phys. 2002, 74, 47. [CrossRef]

43. Montoya, J.M.; Solé, R.V. Small world patterns in food webs. J. Theor. Biol. 2002, 214, 405-412. [CrossRef] [PubMed]

44. Pond, T.; Magsarjav, S.; South, T.; Mitchell, L.; Bagrow, J.P. Complex contagion features without social reinforcement in a model of social information flow. Entropy 2020, 22, 265. [CrossRef]

45. Iannelli, F.; Koher, A.; Brockmann, D.; Hövel, P.; Sokolov, I.M. Effective distances for epidemics spreading on complex networks. Phys. Rev. E 2017, 95, 012313. [CrossRef] [PubMed] 
46. Liu, W.; Liu, C.; Yang, Z.; Liu, X.; Zhang, Y.; Wei, Z. Modeling the propagation of mobile malware on complex networks. Commun. Nonlinear Sci. Numer. Simul. 2016, 37, 249-264. [CrossRef]

47. Ding, L.; Liu, S.Y.; Yang, Q.; Xu, X.K. Uncovering the Dependence of Cascading Failures on Network Topology by Constructing Null Models. Entropy 2019, 21, 1119. [CrossRef]

48. Dobson, I.; Carreras, B.A.; Lynch, V.E.; Newman, D.E. Complex systems analysis of series of blackouts: Cascading failure, critical points, and self-organization. Chaos: Interdiscip. J. Nonlinear Sci. 2007, 17, 026103. [CrossRef] [PubMed]

49. $\mathrm{Fu}, \mathrm{X}$; $\mathrm{Li}, \mathrm{W}$. Cascading failures of wireless sensor networks. In Proceedings of the 11th IEEE International Conference on Networking, Sensing and Control, Miami, FL, USA, 7-9 April 2014; pp. 631-636.

50. Cui, L.; Kumara, S.; Albert, R. Complex networks: An engineering view. Circuits Syst. Mag. IEEE 2010, 10, 10-25. [CrossRef]

51. Crucitti, P.; Latora, V.; Marchiori, M. Model for cascading failures in complex networks. Phys. Rev. E 2004, 69, 045104. [CrossRef] [PubMed]

52. Kinney, R.; Crucitti, P.; Albert, R.; Latora, V. Modeling cascading failures in the North American power grid. Eur. Phys. J. Condens. Matter Complex Syst. 2005, 46, 101-107. [CrossRef]

53. Barthélemy, M. Spatial networks. Phys. Rep. 2011, 499, 1-101. [CrossRef]

54. Bashan, A.; Berezin, Y.; Buldyrev, S.V.; Havlin, S. The extreme vulnerability of interdependent spatially embedded networks. Nat. Phys. 2013, 9, 667-672.

55. Barthelemy, M. Morphogenesis of spatial networks; Springer: New York, NY, USA, 2018.

56. Zhao, J.; Li, D.; Sanhedrai, H.; Cohen, R.; Havlin, S. Spatio-temporal propagation of cascading overload failures in spatially embedded networks. Nat. Commun. 2016, 7, 1-6. [CrossRef]

57. Penrose, M. Random Geometric Graphs; Oxford University Press: Cambridge, UK, 2003; Volume 5.

58. Kenniche, H.; Ravelomananana, V. Random Geometric Graphs as model of wireless sensor networks. In Proceedings of the 2010 The 2nd International Conference on Computer and Automation Engineering (ICCAE), Singapore, 26-28 February 2010; Volume 4, pp. 103-107.

59. Nemeth, G.; Vattay, G. Giant clusters in random ad hoc networks. Phys. Rev. E 2003, 67, 036110. [CrossRef]

60. Gong, L.; Tong, P. von Neumann entropy and localization-delocalization transition of electron states in quantum small-world networks. Phys. Rev. E 2006, 74, 056103. [CrossRef]

61. Umeyama, T.; Igarashi, K.; Sasada, D.; Tamai, Y.; Ishida, K.; Koganezawa, T.; Ohtani, S.; Tanaka, K.; Ohkita, H.; Imahori, H. Efficient light-harvesting, energy migration, and charge transfer by nanographene-based nonfullerene small-molecule acceptors exhibiting unusually long excited-state lifetime in the film state. Chem. Sci. 2020, 11, 3250-3257. [CrossRef]

62. Biamonte, J.; Faccin, M.; De Domenico, M. Complex networks from classical to quantum. Commun. Phys. 2019, 2, 1-10. [CrossRef]

63. Sánchez-Burillo, E.; Duch, J.; Gómez-Gardenes, J.; Zueco, D. Quantum navigation and ranking in complex networks. Sci. Rep. 2012, 2, 605. [CrossRef] [PubMed]

64. Venegas-Andraca, S.E. Quantum walks: A comprehensive review. Quantum Inf. Process. 2012, 11, 1015-1106. [CrossRef]

65. Masuda, N.; Porter, M.A.; Lambiotte, R. Random walks and diffusion on networks. Phys. Rep. 2017, 716, 1-58. [CrossRef]

66. Susskind, L.; Friedman, A. Quantum Mechanics: The Theoretical Minimum; Penguin Books: London, UK, 2015.

67. Ritter, S.; Nölleke, C.; Hahn, C.; Reiserer, A.; Neuzner, A.; Uphoff, M.; Mücke, M.; Figueroa, E.; Bochmann, J.; Rempe, G. An elementary quantum network of single atoms in optical cavities. Nature 2012, 484, 195. [CrossRef]

68. Mülken, O.; Dolgushev, M.; Galiceanu, M. Complex quantum networks: From universal breakdown to optimal transport. Phys. Rev. E 2016, 93, 022304. [CrossRef]

69. Faccin, M.; Migdał, P.; Johnson, T.H.; Bergholm, V.; Biamonte, J.D. Community detection in quantum complex networks. Phys. Rev. X 2014, 4, 041012. [CrossRef]

70. Mohseni, M.; Rebentrost, P.; Lloyd, S.; Aspuru-Guzik, A. Environment-assisted quantum walks in photosynthetic energy transfer. J. Chem. Phys. 2008, 129, 11B603. [CrossRef]

71. Mülken, O.; Blumen, A. Efficiency of quantum and classical transport on graphs. Phys. Rev. E 2006, 73, 066117. [CrossRef]

72. Mülken, O.; Blumen, A. Continuous-time quantum walks: Models for coherent transport on complex networks. Phys. Rep. 2011, 502, 37-87. [CrossRef]

73. Darázs, Z.; Kiss, T. Pólya number of the continuous-time quantum walks. Phys. Rev. A 2010, 81, 062319. [CrossRef]

74. Mülken, O.; Volta, A.; Blumen, A. Asymmetries in symmetric quantum walks on two-dimensional networks. Phys. Rev. A 2005, 72, 042334. [CrossRef]

75. Agliari, E.; Blumen, A.; Mülken, O. Dynamics of continuous-time quantum walks in restricted geometries. J. Phys. Math. Theor. 2008, 41, 445301. [CrossRef]

76. Mülken, O.; Bierbaum, V.; Blumen, A. Coherent exciton transport in dendrimers and continuous-time quantum walks. J. Chem. Phys. 2006, 124, 124905. [CrossRef] [PubMed]

77. Agliari, E.; Blumen, A.; Muelken, O. Quantum-walk approach to searching on fractal structures. Phys. Rev. A 2010, 82, 012305 . [CrossRef]

78. Blumen, A.; Bierbaum, V.; Mülken, O. Coherent dynamics on hierarchical systems. Phys. Stat. Mech. Its Appl. 2006, 371, 10-15. [CrossRef]

79. Mülken, O.; Blumen, A. Slow transport by continuous time quantum walks. Phys. Rev. E 2005, 71, 016101. [CrossRef] 
80. Xu, X.P.; Li, W.; Liu, F. Coherent transport on Apollonian networks and continuous-time quantum walks. Phys. Rev. E 2008, 78, 052103. [CrossRef]

81. Xu, X.; Liu, F. Coherent exciton transport on scale-free networks. New J. Phys. 2008, 10, 123012. [CrossRef]

82. Mülken, O.; Pernice, V.; Blumen, A. Quantum transport on small-world networks: A continuous-time quantum walk approach. Phys. Rev. E 2007, 76, 051125. [CrossRef]

83. Salimi, S. Continuous-time quantum walks on star graphs. Ann. Phys. 2009, 324, 1185-1193. [CrossRef]

84. Anishchenko, A.; Blumen, A.; Mülken, O. Enhancing the spreading of quantum walks on star graphs by additional bonds. Quantum Inf. Process. 2012, 11, 1273-1286. [CrossRef]

85. Kulvelis, N.; Dolgushev, M.; Mülken, O. Universality at breakdown of quantum transport on complex networks. Phys. Rev. Lett. 2015, 115, 120602. [CrossRef] [PubMed]

86. Watts, D.J.; Strogatz, S.H. Collective dynamics of "small-world" networks. Nature 1998, 393, 440-442. [CrossRef] [PubMed]

87. Caldarelli, G.; Vespignani, A. Large Scale Structure and Dynamics of Complex Networks: From Information Technology to Finance and Natural Science; World Scientific: Singapore, 2007; Volume 2.

88. Newman, M.E.; Watts, D.J. Renormalization group analysis of the small-world network model. Phys. Lett. A 1999, 263, 341-346. [CrossRef]

89. Martinez-Mendoza, A.; Alcazar-López, A.; Méndez-Bermúdez, J. Scattering and transport properties of tight-binding random networks. Phys. Rev. E 2013, 88, 012126. [CrossRef]

90. Datta, S. Electronic Transport in Mesoscopic Systems; Cambridge University Press: Cambridge, UK, 1997.

91. Mandl, F. Quantum Mechanics; John Wiley \& Sons: Hoboken, NJ, USA, 1992.

92. Galindo, A.; Pascual, P. Quantum Mechanics I; Springer Science \& Business Media: Berlin/Heidelberg, Germany, 2012.

93. Ballentine, L.E. Quantum Mechanics: A Modern Development; World Scientific Publishing Company: Singapore, 2014.

94. Cohen-Tannoudji, C.; Diu, B.; Laloe, F.; Dui, B. Quantum Mechanics; Wiley-Interscience: Hoboken, NJ, USA, 2006.

95. Farhi, E.; Gutmann, S. Quantum computation and decision trees. Phys. Rev. A 1998, 58, 915. [CrossRef]

96. Faccin, M.; Johnson, T.; Biamonte, J.; Kais, S.; Migdał, P. Degree distribution in quantum walks on complex networks. Phys. Rev. $X$ 2013, 3, 041007. [CrossRef]

97. Mülken, O.; Blumen, A. From Continuous-Time Random Walks to Continuous-Time Quantum Walks: Disordered Networks. In Nonlinear Phenomena in Complex Systems: From Nano to Macro Scale; Springer: Berlin/Heidelberg, Germany, 2014 ; pp. $189-197$.

98. Darázs, Z.; Anishchenko, A.; Kiss, T.; Blumen, A.; Mülken, O. Transport properties of continuous-time quantum walks on Sierpinski fractals. Phys. Rev. E 2014, 90, 032113. [CrossRef]

99. Anishchenko, A.; Blumen, A.; Muelken, O. Geometrical aspects of quantum walks on random two-dimensional structures. Phys. Rev. E 2013, 88, 062126. [CrossRef]

100. Ray, R.K. Solving Quantum Random Walker Using Steepest Entropy Ascent Ansatz: A Pathway Towards Typicality. arXiv 2019, arXiv:1907.04548.

101. Gualtieri, V.; Benedetti, C.; Paris, M.G. Quantum-classical dynamical distance and quantumness of quantum walks. Phys. Rev. A 2020, 102, 012201. [CrossRef]

102. Mülken, O.; Blumen, A. Spacetime structures of continuous-time quantum walks. Phys. Rev. E 2005, 71, 036128. [CrossRef]

103. Stevanovic, D. Applications of graph spectra in quantum physics. In Selected Topics in Applications of Graph Spectra; Mathematical Institute of the Serbian Academy of Sciences and Arts: Belgrade, Serbia, 2011; pp. 85-111.

104. Lee, D.; Kahng, B.; Cho, Y.; Goh, K.I.; Lee, D.S. Recent advances of percolation theory in complex networks. J. Korean Phys. Soc. 2018, 73, 152-164. [CrossRef]

105. Luque, A.; Martí, A.; Stanley, C. Understanding intermediate-band solar cells. Nat. Photonics 2012, 6, 146-152. [CrossRef]

106. Luque, A.; Martí, A. Increasing the efficiency of ideal solar cells by photon induced transitions at intermediate levels. Phys. Rev. Lett. 1997, 78, 5014. [CrossRef]

107. Shockley, W.; Queisser, H.J. Detailed balance limit of efficiency of p-n junction solar cells. J. Appl. Phys. 1961, 32, 510-519. [CrossRef]

108. Luque, A.; Linares, P.; Antolín, E.; Ramiro, I.; Farmer, C.; Hernández, E.; Tobías, I.; Stanley, C.; Martí, A. Understanding the operation of quantum dot intermediate band solar cells. J. Appl. Phys. 2012, 111, 044502. [CrossRef]

109. López, E.; Datas, A.; Ramiro, I.; Linares, P.; Antolín, E.; Artacho, I.; Martí, A.; Luque, A.; Shoji, Y.; Sogabe, T.; et al. Demonstration of the operation principles of intermediate band solar cells at room temperature. Sol. Energy Mater. Sol. Cells 2016, 149, 15-18. [CrossRef]

110. Luque, A.; Martí, A.; López, N.; Antolín, E.; Cánovas, E.; Stanley, C.; Farmer, C.; Caballero, L.; Cuadra, L.; Balenzategui, J. Experimental analysis of the quasi-Fermi level split in quantum dot intermediate-band solar cells. Appl. Phys. Lett. 2005, 87, 083505. [CrossRef]

111. Martí, A.; Antolín, E.; Stanley, C.; Farmer, C.; López, N.; Díaz, P.; Cánovas, E.; Linares, P.; Luque, A. Production of photocurrent due to intermediate-to-conduction-band transitions: A demonstration of a key operating principle of the intermediate-band solar cell. Phys. Rev. Lett. 2006, 97, 247701. [CrossRef] [PubMed]

112. Datas, A.; López, E.; Ramiro, I.; Antolín, E.; Martí, A.; Luque, A.; Tamaki, R.; Shoji, Y.; Sogabe, T.; Okada, Y. Intermediate band solar cell with extreme broadband spectrum quantum efficiency. Phys. Rev. Lett. 2015, 114, 157701. [CrossRef] [PubMed] 
113. Wełna, M.; Żelazna, K.; Létoublon, A.; Cornet, C.; Kudrawiec, R. Stability of the intermediate band energy position upon temperature changes in GaNP and GaNPAs. Sol. Energy Mater. Sol. Cells 2019, 196, 131-137. [CrossRef]

114. Jiang, J.; Zhou, W.; Xue, Y.; Ning, H.; Liang, X.; Zhou, W.; Guo, J.; Huang, D. Intermediate band insertion by group-IIIA elements alloying in a low cost solar cell absorber CuYSe2: A first-principles study. Phys. Lett. A 2019, 383, 1972-1976. [CrossRef]

115. Ramiro, I.; Martí, A.; Antolín, E.; Luque, A. Review of experimental results related to the operation of intermediate band solar cells. IEEE J. Photovoltaics 2014, 4, 736-748. [CrossRef]

116. Martí, A.; Luque, A. Fundamentals of intermediate band solar cells. In Next, Generation of Photovoltaics; Springer: Berlin/Heidelberg, Germany, 2012; pp. 209-228.

117. Marrón, D.F. Thin-Film Technology in Intermediate Band Solar Cells: Advanced Concepts for Chalcopyrite Solar Cells. In Next, Generation of Photovoltaics; Springer: Berlin/Heidelberg, Germany, 2012; pp. 277-307.

118. Foxon, C.T.; Novikov, S.V.; Campion, R.P. InGaN Technology for IBSC Applications. In Next, Generation of Photovoltaics; Springer: Berlin/Heidelberg, Germany, 2012; pp. 309-319.

119. Olea, J.; Pastor, D.; Luque, M.T.; Mártil, I.; Díaz, G.G. Ion implant technology for intermediate band solar cells. In Next, Generation of Photovoltaics; Springer: Berlin/Heidelberg, Germany, 2012; pp. 321-346.

120. Antolín, E.; Martí, A.; Olea, J.; Pastor, D.; González-Díaz, G.; Mártil, I.; Luque, A. Lifetime recovery in ultrahighly titanium-doped silicon for the implementation of an intermediate band material. Appl. Phys. Lett. 2009, 94, 042115. [CrossRef]

121. Persans, P.D.; Berry, N.E.; Recht, D.; Hutchinson, D.; Peterson, H.; Clark, J.; Charnvanichborikarn, S.; Williams, J.S.; DiFranzo, A.; Aziz, M.J.; et al. Photocarrier lifetime and transport in silicon supersaturated with sulfur. Appl. Phys. Lett. 2012, 101, 111105. [CrossRef]

122. Luque, A.; Martí, A.; Antolín, E.; Tablero, C. Intermediate bands versus levels in non-radiative recombination. Phys. Condens. Matter 2006, 382, 320-327. [CrossRef]

123. Mott, N. Metal-insulator transition. Rev. Mod. Phys. 1968, 40, 677. [CrossRef]

124. Mott, N.F.; Davis, E.A. Electronic Processes in Non-Crystalline Materials; Oxford University Press: Oxford, UK, 2012.

125. Scholes, G.D.; Fleming, G.R.; Olaya-Castro, A.; Van Grondelle, R. Lessons from nature about solar light harvesting. Nat. Chem. 2011, 3, 763-774. [CrossRef]

126. Kundu, S.; Patra, A. Nanoscale strategies for light harvesting. Chem. Rev. 2017, 117, 712-757. [CrossRef] [PubMed]

127. Sarovar, M.; Ishizaki, A.; Fleming, G.R.; Whaley, K.B. Quantum entanglement in photosynthetic light-harvesting complexes. Nat. Phys. 2010, 6, 462-467. [CrossRef]

128. Nalbach, P.; Thorwart, M. Enhanced quantum efficiency of light-harvesting in a biomolecular quantum "steam engine". Proc. Natl. Acad. Sci. USA 2013, 110, 2693-2694. [CrossRef] [PubMed]

129. Jiang, Y.; McNeill, J. Light-harvesting and amplified energy transfer in conjugated polymer nanoparticles. Chem. Rev. 2017, 117, 838-859. [CrossRef] [PubMed]

130. Yang, P.Y.; Cao, J. Steady-state analysis of light-harvesting energy transfer driven by incoherent light: From dimers to networks. J. Phys. Chem. Lett. 2020, 11, 7204-7211. [CrossRef] [PubMed] 